\title{
Coulomb-blockade oscillations in disordered quantum wires
}

\author{
A. A. M. Staring, H. van Houten, and C. W. J. Beenakker \\ Philips Research Laboratories, 5600 JA Eindhoven, The Netherlands \\ C. T. Foxon \\ Philips Research Laboratories, Redhill RH1 5HA, United Kingdom
}

(Received 15 July 1991)

\begin{abstract}
The conductance of narrow wires, defined by a split-gate technique in the two-dimensional electron gas in a modulation-doped $\mathrm{GaAs}-\mathrm{Al}_{x} \mathrm{Ga}_{1-x}$ As heterostructure, is studied experimentally as a function of gate voltage, temperature, and magnetic field. Both intentionally (Be doped) and unintentionally disordered wires are investigated. Periodic conductance oscillations as a function of gate voltage are found in both systems, in the regime where only a few hundred electrons are present in the wire. The dominant oscillations are very regularly spaced, with a period that is quite insensitive to a strong magnetic field, and persist up to a few kelvin. A strong magnetic field is found to enhance the amplitude of the oscillations up to values approaching $e^{2} / h$. The experimental data are analyzed in terms of a theory for Coulomb-blockade oscillations in the conductance of a quantum dot in the regime of comparable level spacing $\Delta E$ and charging energy $e^{2} / C$, based on the assumed presence of a conductance-limiting segment in the wire. Good agreement with the experiment is obtained for the temperature dependence of the oscillations, using physically reasonable parameter values. At low temperatures, a crossover from the classical regime $k_{B} T \gtrsim \Delta E$ to the quantum regime $k_{B} T \lesssim \Delta E$ is found. The appearance of additional periodicities and the onset of irregular oscillations at very low temperatures in some of the wires are attributed to the presence of multiple segments. No magnetoconductance oscillations are observed, in support of the recently predicted Coulomb blockade of the Aharonov-Bohm effect.
\end{abstract}

\section{INTRODUCTION}

The phenomenon investigated experimentally in this paper was first observed by Scott-Thomas et al. ${ }^{1}$ They discovered that at low temperatures a narrow disordered channel in a Si inversion layer may exhibit strikingly regular conductance oscillations as a function of the voltage on the gates used to define the channel. This is in contrast to the aperiodic conductance fluctuations usually observed in such structures. ${ }^{2}$ The period of the oscillations differed from device to device, and did not correlate with the channel length. Based on estimates of the sample parameters, it was concluded that each period corresponds to the addition of a single electron to a conductance-limiting segment in the narrow channel. In order to explain thcir observations, Scott-Thomas et $a l^{1}$ originally suggested that a charge-density wave or "Wigner crystal" was formed. From a model due to Larkin and Lee, ${ }^{3}$ and Lee and Rice, ${ }^{4}$ they inferred that this would lead to a thermally activated conductance because of the pinning of the charge-density wave by impurities in the narrow channel. The activation energy would be dctermined by the most strongly pinned segment of the crystal, and periodic oscillations in the conductance as a function of gate voltage or electron density would reflect the condition that an integer number of electrons is contained between the two impurities delimiting that specific segment.

As an alternative explanation, two of us have proposed that the effect is a manifestation of Coulomb-blockade oscillatrons in a semiconductor nanostructure. ${ }^{5}$ In the discussion of our experimental results, we limit ourselves to a comparison with the Coulomb-blockade model, for which the theory has now been worked out. ${ }^{6-8}$ A discussion of the Wigner-crystal model has been given in Refs. 9 and 10 . The conclusion reached in the present paper is that the Coulomb-blockade model does provide an adequate and consistent description of our experiments. In a low-density quantum wire with weak disorder (no tunnel barriers), however, a Wigner-crystal may well be an appropriate description of the ground state. ${ }^{11}$

The Wigner crystal is a manifestation of long-range order neglected in the theory of Coulomb-blockade oscillations. However, both the Coulomb blockade and the Wigner-crystal models have in common that electronelectron interactions play a central role. In contrast, some authors have argued that resonant tunneling of noninteracting electrons can explain the periodicity of the observed conductance oscillations. ${ }^{12,13}$ One cannot easily discriminate between these models on the basis of the periodicity of the oscillations. Conductance oscillations due to resonant tunneling through nondegenerate levels as well as Coulomb-blockade oscillations both have a periodicity corresponding to the addition of a single electron to the confined region. Other considerations are necessary to demonstrate the inadequacy of a model based on resonant tunneling of noninteracting electrons. The most important of these are the large activation energy 
of the minima (exceeding the average single-electron level spacing $\Delta E$ ), the absence of spin splitting of the peaks in a magnetic field, and the absence of magnetoconductance oscillations. These considerations will be discussed in detail in this paper.

Our experimental work has consisted of a study of the conductance of disordered quantum wires defined by a split-gate technique in the two-dimensional electron gas (2DEG) of a GaAs- $\mathrm{Al}_{x} \mathrm{Ga}_{1-x}$ As heterostructure. We have investigated the effects of temperature and magnetic field on the conductance as a function of gate voltage, as well as the magnetoconductance and the Hall resistance in a cross-shaped narrow channel geometry. In addition, we have varied the channel length, and the degree of disorder. Some of our results have been published previously. ${ }^{14}$ It is the purpose of this paper to give a. more complete account of our experimental work, and to present a quantitative comparison with the theor ${ }^{6-8}$ for Coulomb-blockade oscillations in the size-quantized regime characteristic of semiconductor nanostructures. ${ }^{15}$

Other observations of the effect have recently been reported by Field et al. ${ }^{9}$ in a narrow channel in a $2 \mathrm{D}$ hole gas in $\mathrm{Si}$, by Meirav et al. ${ }^{16}$ in a narrow electron gas channel in an inverted $\mathrm{GaAs}-\mathrm{Al}_{x} \mathrm{Ga}_{1-x} \mathrm{As}$ heterostructure, and by De Graaf et al. ${ }^{17}$ in a very short split-gate channel (or point contact) in a $\mathrm{Si}$ inversion layer. In addition, Coulomb-blockade oscillations have been observed in the conductance of a quantum dot by several groups. ${ }^{18-22}$ This work has been reviewed recently, ${ }^{23}$ and will not be discussed here.

This paper is organized as follows. The split-gate quantum wires used in our study are described in Sec. II. An overview of the experimental results is given in Sec. III. We find a rich and complex behavior, with variations from device to device, reflecting the mesoscopic nature of disordered quantum wires. The most characteristic aspects of our observations, however, are representative of all devices that show the conductance oscillations. The period of the oscillations as a function of gate voltage is explained in terms of a theory for Coulomb-blockade oscillations in Sec. IV A, using an equivalent circuit to model the electrostatics of the problem. We can account for the temperature dependence of the line shape of the oscillations as well, as is discussed in Sec. IV B. The effects of multiple segments in the wire are discussed in Sec. IV C. Finally, we discuss in Sec. V those aspects of the experimental results that are less well understood, and conclude.

\section{SPLIT-GATE QUANTUM WIRES}

Our experimental results for the conductance of quasione-dimensional channels have been obtained using narrow wires, defined by a split-gate technique in the 2DEG in a modulation doped $\mathrm{GaAs}-\mathrm{Al}_{x} \mathrm{Ga}_{1-x}$ As heterostructure. By adjusting the negative gate voltage (applied between the gate on top of the heterostructure and an Ohmic contact to the 2DEG), the channel width $W$ can be controlled in a range from definition (where $W \approx$ $W_{\text {ltth }}$, the lithographic width) to pinch off (where $W$ is close to zero). In the regime of interest, which is that close to pinch off, both the electron concentration per unit length and the channel width vary approximately linearly with gate voltage. ${ }^{24}$

Starting point for the fabrication of our samples is a GaAs- $\mathrm{Al}_{x} \mathrm{Ga}_{1-x} \mathrm{As}$ heterostructure, which consists of a sequence of layers grown on top of a semi-insulating GaAs substrate by molecular-beam epitaxy. The first layer is a thick buffer layer of pure GaAs. The 2DEG is formed at the interface of this layer with an $\mathrm{Al}_{0}{ }_{33} \mathrm{Ga}_{0}{ }_{67} \mathrm{As}$ layer grown on top of it. The latter consists of a 20-nm-thick spacer layer of pure $\mathrm{Al}_{0}{ }_{33} \mathrm{Ga}_{0}{ }_{67} \mathrm{As}$, which serves to separate the electrons from their parent donors in order to increase their mobility, and a $40-\mathrm{nm}$-thick $\mathrm{Al}_{0}{ }_{33} \mathrm{Ga}_{0}{ }_{67} \mathrm{As}$ layer doped with $\mathrm{Si}$ at a concentration of $1.33 \times 10^{18}$ $\mathrm{cm}^{-3}$. Finally, the heterostructure is capped by a 20 nm-thick undoped GaAs layer.

We have used two sets of samples. In one set (designated by $D$ in Table I), a planar doping layer of $\mathrm{Be}$ impurities with a sheet concentration of $2 \times 10^{10} \mathrm{~cm}^{-2}$ was incorporated in the buffer layer during growth, at $25 \AA$ below the heterointerface. This was done in order to introduce strongly repulsive scattering centers in the 2DEG (Be is an acceptor in GaAs). Such scattering centers may act as tunnel barriers in a narrow channel in the 2 DEG. ${ }^{5}$ The other set of samples (designated by $U$ ) was undoped, but was nevertheless disordered as wcll, due to random fluctuations in the distribution of the ionized donors in the $\mathrm{Al}_{x} \mathrm{Ga}_{1-x}$ As layer. ${ }^{25}$

In the wide regions, the Be-doped samples had an elastic mean free path $l_{e} \approx 0.7 \mu \mathrm{m}$, deduced from the conductivity at $T=4.2 \mathrm{~K}$ and the electron sheet density $n_{s}=2.9 \times 10^{11} \mathrm{~cm}^{-2}$. For the undoped samples these values were $l_{e}=3.9 \mu \mathrm{m}$ and $n_{s}=3.0 \times 10^{11} \mathrm{~cm}^{-2}$. This mean free path does not describe the transport in the quantum wires near pinch off, when the conductance is limited by a small number of accidentally strong scattering centers. These are due to negatively charged $\mathrm{Be}$

TABLE I. Channel length and period of the conductance oscillations.

\begin{tabular}{ccc}
\hline \hline Channel $^{\mathrm{a}, \mathrm{b}}$ & $\begin{array}{c}\text { Length } \\
(\mu \mathrm{m})\end{array}$ & $\begin{array}{c}\text { Period }^{\mathrm{c}} \\
(\mathrm{mV})\end{array}$ \\
\hline$D 1$ & 4.4 & 2.7 \\
$D 2$ & 6.2 & 2.1 \\
$D 3$ & 6.3 & 2.2 \\
$U 1$ & 0.5 & \\
$U 2$ & 6.2 & 1.0 \\
$U 3$ & 16.7 & 2.3 \\
\hline \hline
\end{tabular}

a The $D$ channels are intentionally disordered by means of a planar doping layer of Be near the heterointerface in the GaAs layer. The $U$ channels are unintentionally disordered.

${ }^{\mathrm{b}}$ Channel $D 1$ is the right section and channel $D 3$ is the middle section of a miniature Hall bar [see Fig. 1(b)].

c The period of the oscillations is given for $T=1.5 \mathrm{~K}$ and $B=0$, except for channel $U 2(T=50 \mathrm{mK}$ and $B=0)$ and for channel $D 3(T=50 \mathrm{mk}$ and $B=5 \mathrm{~T})$. No oscillations were observed in the shortest channel $U 1$. 
acceptors close to the 2DEG, and due to statistical fluctuations in the distribution of the remote ionized donors in the $\mathrm{Al}_{x} \mathrm{Ga}_{1-x}$ As layer. The resulting variations in the electrostatic potential are enhanced in a narrow channel because of the reduced screening. Near pinch off, the channel breaks up into a small number of segments separated by potential barriers formed by such scattering centers. This is inferred from our experimental results, and is supported by model calculations of Nixon and Davies, ${ }^{25}$ in which the random positions of the remote ionized donors are taken into account.

The fabrication of the samples proceeds as follows. First, the heterostructure is mesa-etched into a rectangular shape, and twelve alloyed $\mathrm{Au}-\mathrm{Ge}-\mathrm{Ni}$ Ohmic contacts are formed along its edges. Then, a pattern of six TiAu gate electrodes is defined in a two-step process, using optical lithography for the coarse parts and electronbeam lithography for the fine details. These gates can be controlled independently. Figure 1 shows scanning electron micrographs of the two narrow-channel geometries studied. When negatively biased, the gates (light lines) subdivide the 2DEG into six wide regions (underneath the dark areas), which are connected by narrow channels. Two Ohmic contacts are attached to each of these wide regions. The first geometry [Fig. 1(a)] consists of a set of five narrow channels on a single sample (each of
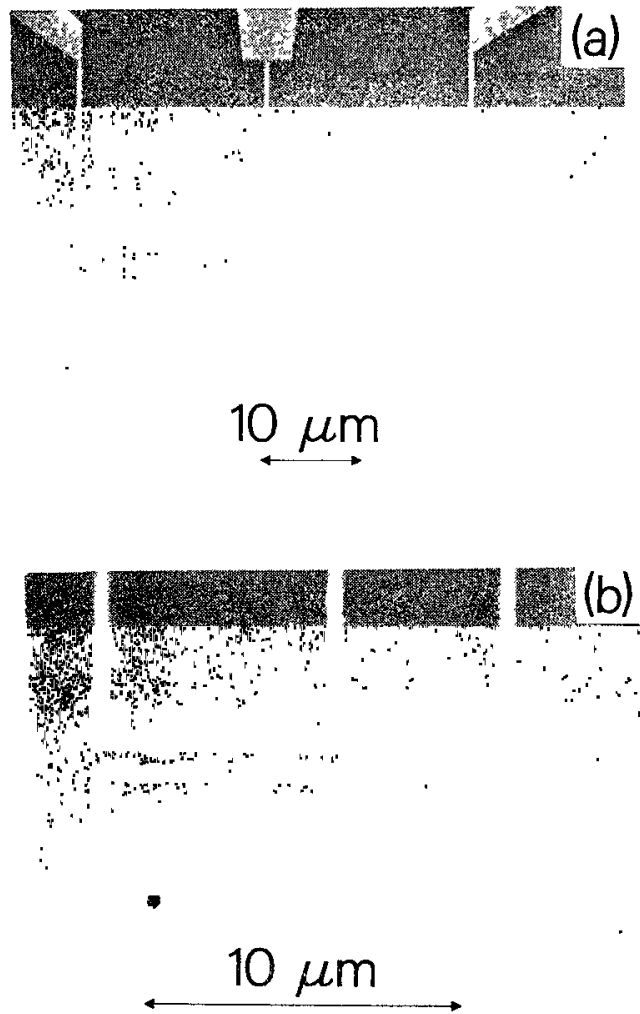

FIG. 1. Scanning electron micrographs of the two splitgate geometries that we have used. The first (a) defines five narrow channels of increasing length, $L=0.5,2.1,6.2,6.2$, and $16.7 \mu \mathrm{m}$, respectively. The second (b) defines a miniature Hall bar, with section lengths $L=4.4,6.3$, and $2.4 \mu \mathrm{m}$ and side probes having a width of $0.5 \mu \mathrm{m}$. For both geometries, the lithographic channel width is $W_{11 \mathrm{~h}}=0.5 \mu \mathrm{m}$. which can be measured independently), while the second [Fig. 1(b)] consists of a miniature Hall bar. At the depletion threshold of the 2DEG directly underneath the gates (about $-0.3 \mathrm{~V}$ ), the narrow channels have approximately the lithographic width $W_{\mathrm{lt} t h}=0.5 \mu \mathrm{m}$. Close to pinch off the channel width $W$ is reduced to about $0.1 \mu \mathrm{m}$, and the electron density $n_{s}$ is reduced by about a factor of 2. (The estimate for $W$ is based on typical lateral depletion widths of $0.2 \mu \mathrm{m} / \mathrm{V},{ }^{15,24,25}$ and that for $n_{s}$ on an extrapolation of the periodicity of the Shubnikov-de Haas oscillations, measured at several gate voltage values.) The length $L$ of the channels varies (see Table I).

One Be-doped sample (not included in Table I), having channels of width $W_{\mathrm{hth}}=1 \mu \mathrm{m}$, was studied as well. The results obtained with these channels wcre similar to those obtained with the narrower channels, except for the pinch-off voltage, which was about twice as large. The periodicity of the dominant oscillations was within the range of values we found in the narrower wires.

\section{EXPERIMENTAL RESULTS}

Primarily, we have performed measurements of the conductance as a function of gate voltage, for a number of quantum wires of different length. The experiments were done over a range of temperatures and magnetic fields. In addition, we have measured the conductance and Hall resistance as a function of magnetic field, at fixed gate voltage. The samples were mounted in the mixing chamber of a dilution refrigerator with a base temperature of $50 \mathrm{mK}$. We employed a magnet capable of generating magnetic fields up to $8 \mathrm{~T}$ perpendicular to the 2DEG. A conventional ac lock-in technique was used to measure the conductance, while the gate voltage (or magnetic field) was swept slowly. In order to ensure linear response, the excitation voltage was kept below $k_{B} T / e$. We have studied the differential conductance also, using dc bias voltages up to a few $\mathrm{mV}$, but in this paper we restrict ourselves to the linear response regime. Experimental data are presented for channels $D 1, D 2$, and $D 3$, which are intentionally disordered by a planar doping layer of $\mathrm{Be}$, and for channels $U 2$ and $U 3$, which are not intentionally disordered.

\section{A. Conductance versus gate voltage: Zero magnetic field}

In Fig. 2 the conductance near pinch off is shown for two Be-doped quantum wires, $D 1$ and $D 2$. At $T=1.5$ $\mathrm{K}$ both channels exhibit well-resolved conductance oscillations, which are perioduc in the gate voltage. The oscillations appear to be superimposed on a background conductance of approximately $0.1 e^{2} / h$, and have a period $\Delta V_{\text {gate }} \approx 2.7 \mathrm{mV}(D 1)$ and $2.1 \mathrm{mV}(D 2)$. As the gate voltage is increased the oscillations disappear gradually. Whercas the two conductance traces are relatively similar at $T=1.5 \mathrm{~K}$, this is not the case at $T=50 \mathrm{mK}$. In channel $D 2$ the oscillations become better resolved at this low temperature, while the period is unchanged and the value of the conductance at the maxima remains ap- 


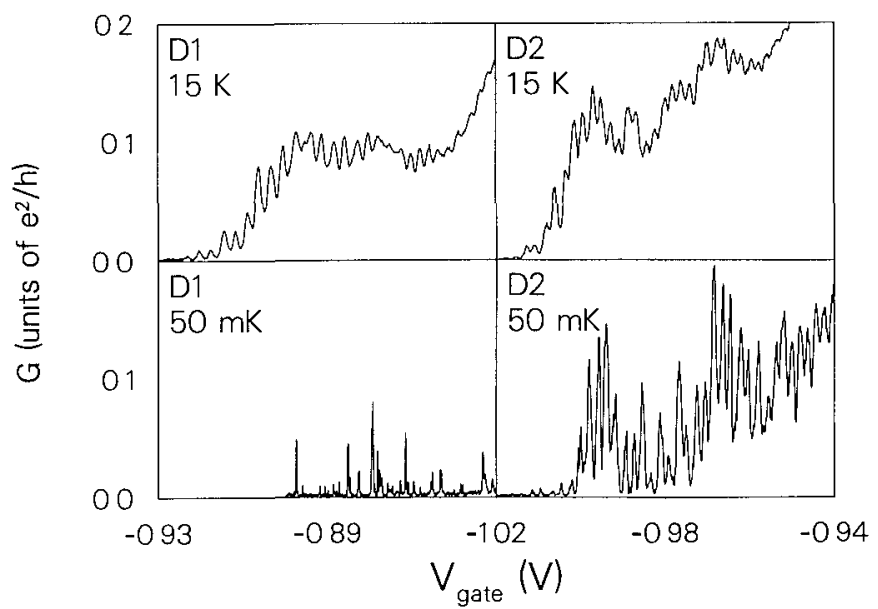

FIG. 2. Two-terminal conductance vs gate voltage of two intentionally disordered narrow channels $(D 1$ and $D 2)$ at $T=$ $1.5 \mathrm{~K}$ and $50 \mathrm{mK}$.

proximately the same. In contrast, the oscillations in channel $D 1$ are suppressed at $50 \mathrm{mK}$, and an irregular pattern of sharp conductance peaks is observed instead.

In Fig. 3 we show a corresponding set of results for two undoped channels, $U 2$ and $U 3$. At $T=1.5 \mathrm{~K}$, the periodic conductance oscillations are observed in channel $U 3$ only $\left(\Delta V_{\text {gate }} \approx 2.3 \mathrm{mV}\right)$. Channel $U 2$ shows a slow conductance modulation instead. Both channels show periodic conductance oscillations as the temperature is decreased to $100 \mathrm{mK}$ ( $\Delta V_{\text {gate }} \approx 1.0 \mathrm{mV}$ for $\left.U 2\right)$. As is the case in channel $D 2$ in Fig. 2, the oscillations in channel $U 3$ become better resolved on lowering the temperature. In addition, a fine structure develops on these peaks, indicative of a higher-frequency oscillation.

The conductance oscillations for channel $U 3$ are shown in more detail in the top panel of $\mathrm{Fig} .4$, for temperatures between 1 and $3 \mathrm{~K}$ (the calculated curves in the bottom panel will be explained in Sec. IV B). Note that both the minima and maxima of the oscillations increase with temperature. At $T=2.5 \mathrm{~K}$ the oscillations are smeared

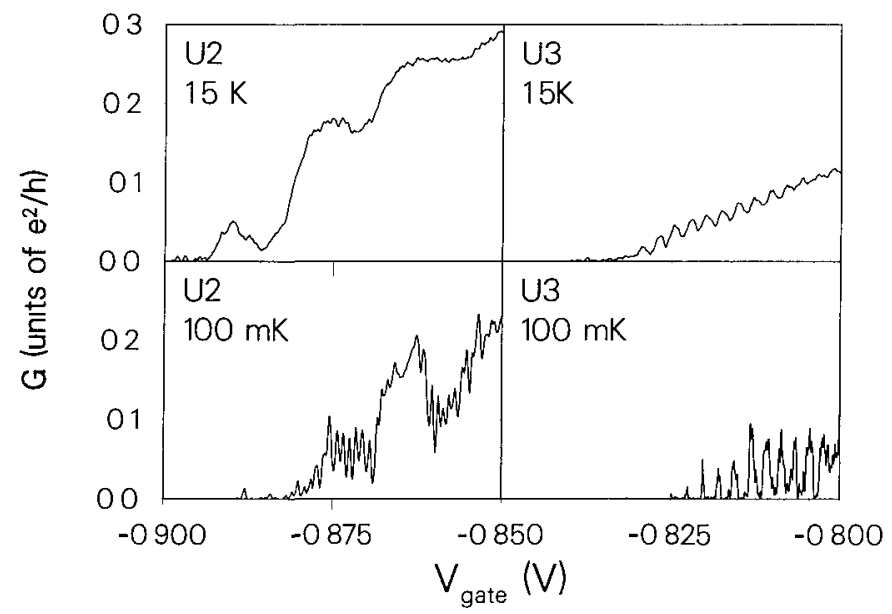

FIG. 3. Two-terminal conductance vs gate voltage of two unintentionally disordered narrow channels ( $U 2$ and $U 3$ ) at $T=1.5 \mathrm{~K}$ and $100 \mathrm{mK}$.

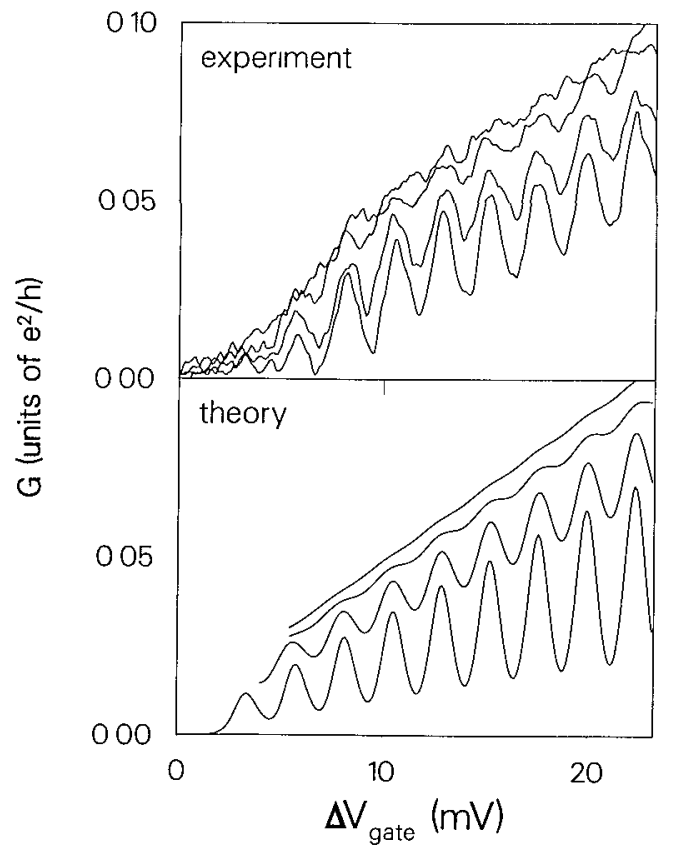

FIG. 4. Top panel: two-terminal conductance vs gate voltage of channel $U 3$ for $T=3.2,2.5,1.6$, and $1 \mathrm{~K}$, from top to bottom. Bottom panel: conductance calculated from Eq. (9) for $e^{2} / C=0.6 \mathrm{meV}, \Delta E=0.1 \mathrm{meV}, \alpha=0.265$, $h \Gamma_{p}^{l, r}=0.027 p E_{p}$, and twofold degeneracy.

out, but can still be resolved.

The results shown in Figs. 2-4 are representative of all the channels we have studied, except for the shortest channel $(U 1, L=0.5 \mu \mathrm{m})$. As evidenced by the conductance, pinch off is typically reached at $-1 \lesssim V_{\text {gate }} \lesssim-0.8$ $V$. Periodic conductance oscillations are observed in most of the channels at temperatures of $1.5 \mathrm{~K}$ or below, with a period varying between 1 and $3 \mathrm{mV}$ for different channels. We did not find systematic differences between the Bedoped channels and the channels which were not intentionally disordered. The period does not correlate with the length of the channel or the degree of disorder (see Table I), and changes within this range when the sample is thermally cycled. The number of successive oscillations observed is between 20 and 50 for most narrow channels. At very low temperatures (below $100 \mathrm{mK}$ ) it is found often that the regular oscillations are replaced by an irregular pattern of sharp conductance peaks.

\section{B. Conductance versus gate voltage: Quantum Hall effect regime}

The various effects of a strong magnetic field on the conductance as a function of gate voltage arc shown in Figs. 5-9 for channels $D 1$ and $D 2$, and in Fig. 10 for channel $U 2$. Figure 5 shows the conductance as a function of gate voltage for channel $D 2$, at four values of the magnetic field. We find that the period of the oscillations is insensitrve to the magnetic field, which is illustrated most clearly by the insets, showing the Fourier spectra of the conductance traces. Each of these exhibits a sharp peak at a $B$-independent frequency of about $450 \mathrm{~V}^{-1}$ (at 


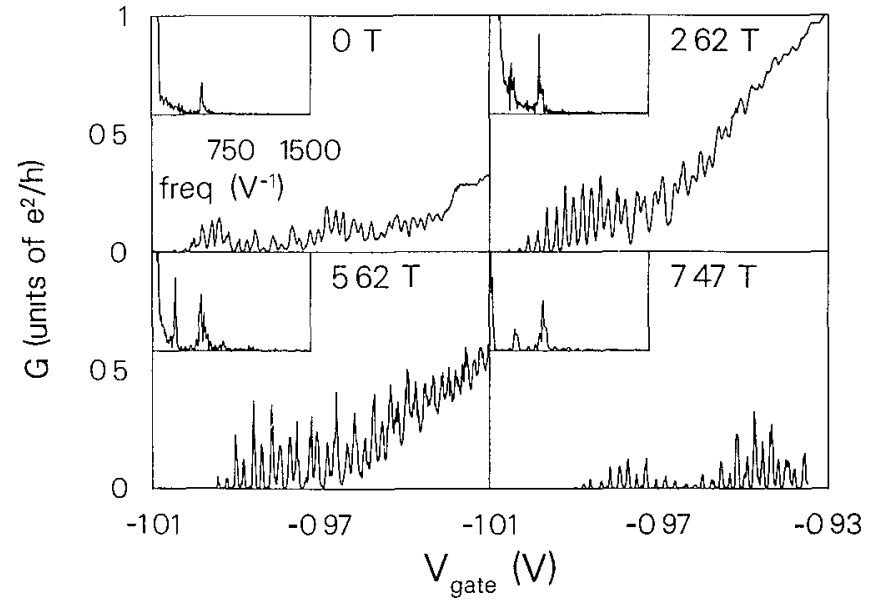

FIG. 5. Two-terminal conductance vs gate voltage of channel $D 2$ at $50 \mathrm{mK}$ in a perpendicular magnetic field. Insets: Fourier spectra of the data. The vertical scale of the Fourier spectra at $B=0$ and $7.47 \mathrm{~T}$ is multiplied by a factor 2.5 .

$B=7.47 \mathrm{~T}$ the frequency has increased by a few percent). The amplitude of the oscillations and the average conductance depend on the magnetic field in a nonmonotonic fashion. As the magnetic field is increased, both the amplitude and average conductance are enhanced above the zero-field values in magnetic fields of intermediate strength (2.62 and $5.62 \mathrm{~T}$ ), followed by a decrease in still stronger fields $(7.47 \mathrm{~T})$. The conductance peaks do not split, not even in our strongest field of $8 \mathrm{~T}$. In this particular channel, however, a second peak emerges in the Fourier spectrum at approximately half the dominant frequency as the magnetic field is increased. This second peak is a result of the amplitude modulation of the peaks in the gate-voltage scan, which is seen most clearly in the trace at $5.62 \mathrm{~T}$, where high- and low-conductance peaks alternate in a doubletlike structure. We do not think that the electron spin is responsible for this effect. Some other channels were found to exhibit more than two peaks in the Fourier spectrum. We attribute these multiple periodicities to the presence of more than one segment in the wire. Finally, we note that with increasing magnetic field pinch-off is reached at loss negative gate voltages, but that the total number of peaks remains approximately constant.

Figure 6 gives the conductance of channel $D 1$ at $T=$ $4.2 \mathrm{~K}(\mathrm{a}), 1.5 \mathrm{~K}(\mathrm{~b})$, and $50 \mathrm{mK}(\mathrm{c})$, at various values of the magnetic field. At $4.2 \mathrm{~K}$ [Fig. $6(\mathrm{a})$ ], the oscillations are almost smeared out in the absence of a magnetic field, and the conductance increases monotonically with gate voltage. Surprisingly, at $B=1.24 \mathrm{~T}$ the oscillations can be observed clearly at this relatively high temperature. The periodic oscillations can be observed best in the traces at $1.5 \mathrm{~K}$ [Fig. 6(b)]. The magnctic-field dependence is similar to that of channel $D 2$, including the insensitivity of the period to the magnetic field, the absence of spin-splitting, and enhancement of the amplitude and average conductance at intermediate field strengths ( $1 \mathrm{~T} \lesssim B \lesssim 5 \mathrm{~T}$ ). In Fig. 2 we have shown that at 50
$\mathrm{mK}$, and in the absence of a magnetic field, the periodic oscillations in channel $D 1$ are suppressed. This is evident in the zero-field trace in Fig. 6(c) as well, where a pattern of irregular conductance peaks is visible, with a typical spacing about five times smaller than the period of the oscillations at $1.5 \mathrm{~K}$. The enhancement of the conductance in fields of intermediate strength is very pronounced at $50 \mathrm{mK}$, where the conductance near $V_{\text {gate }} \approx-0.8 \mathrm{~V}$ ap-
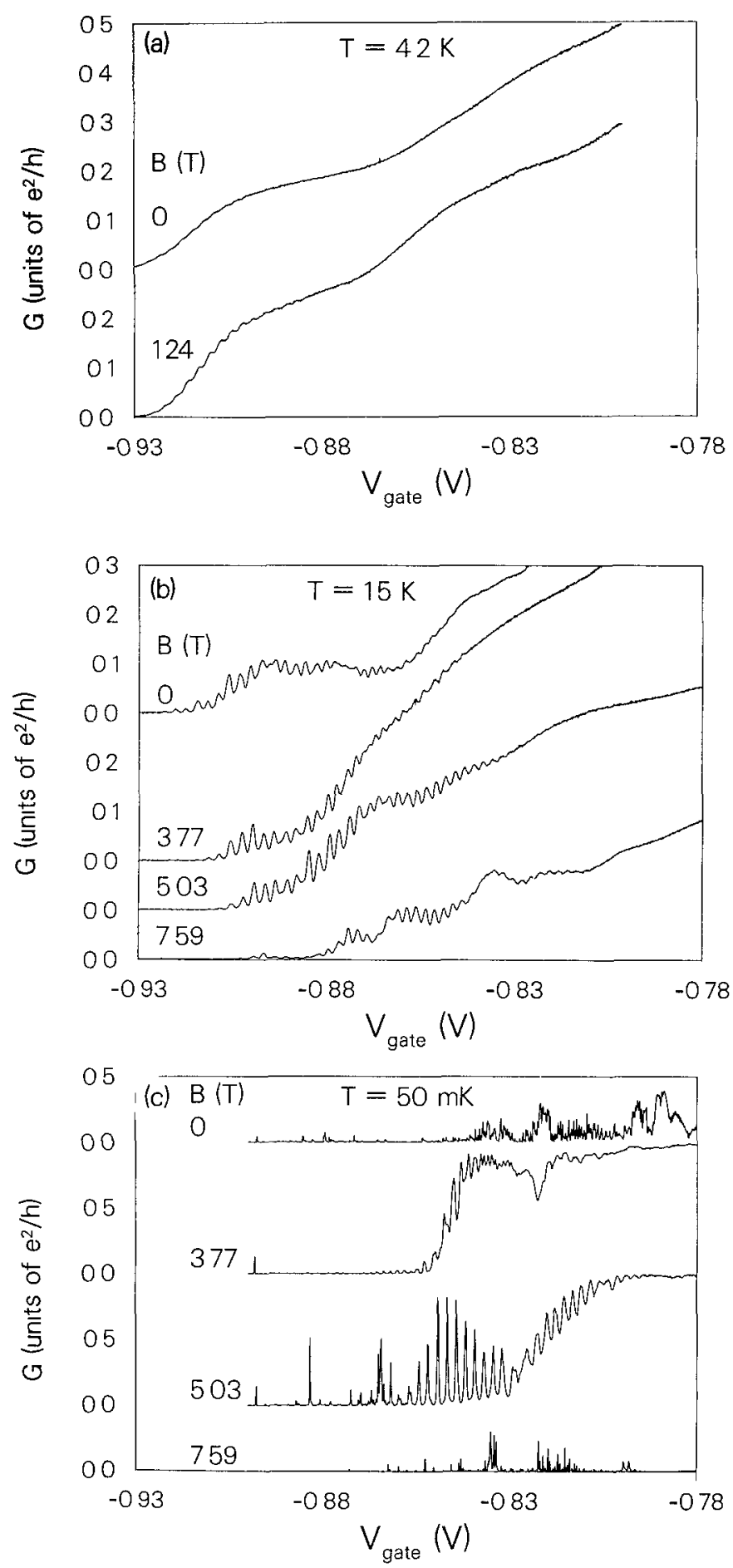

FIG. 6. Two-terminal conductance vs gate voltage of channel $D 1$ in a perpendicular magnetic field. The temperatures ale (a) $4.2 \mathrm{~K}$, (b) $1.5 \mathrm{~K}$, and (c) $50 \mathrm{mK}$. The curves have been offset for clarity. 


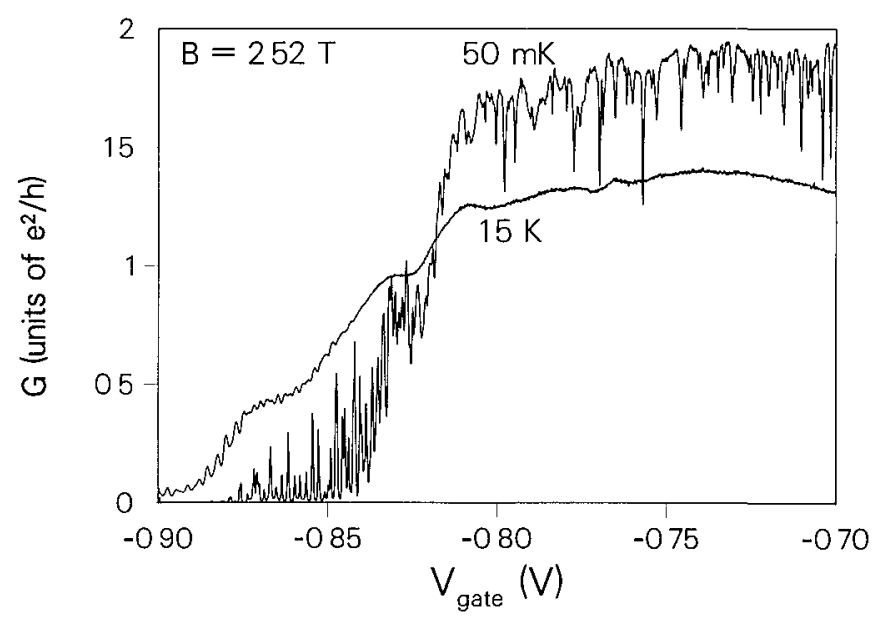

FIG. 7. Two-terminal conductance vs gate voltage of channel $D 1$ at $B=2.52 \mathrm{~T}$, for $T=50 \mathrm{mK}$ and $1.5 \mathrm{~K}$.

proaches the first quantized Hall plateau $\left(G=e^{2} / h\right)$. In the trace at $B=5.03 \mathrm{~T}$ the step region before the $G=e^{2} / h$ plateau exhibits quite pronounced oscillations with the same periodicity as those at $1.5 \mathrm{~K}$, but with an amplitude that is almost equal to $e^{2} / h$. At more negative gate voltages the regularity of the conductance oscillations is lost. This is also the case in stronger magnetic fields.

In Fig. 7 the conductance of channel $D 1$ is shown over a wider range of gate voltage, at $B=2.52 \mathrm{~T}$ and $T=50$ $\mathrm{mK}$ and $1.5 \mathrm{~K}$. At gate voltages below $-0.83 \mathrm{~V}$ the periodic conductance oscillations can be observed in both traces. As the gate voltage is increased beyond $-0.8 \mathrm{~V}$, the conductance at $50 \mathrm{mK}$ is seen to increase up to a value close to the second quantized Hall plateau at $G=2 e^{2} / h$. However, a large number of sharp dips in the conductance are observed in this regime. This structure has vanished completely at $1.5 \mathrm{~K}$, and the conductance plateau

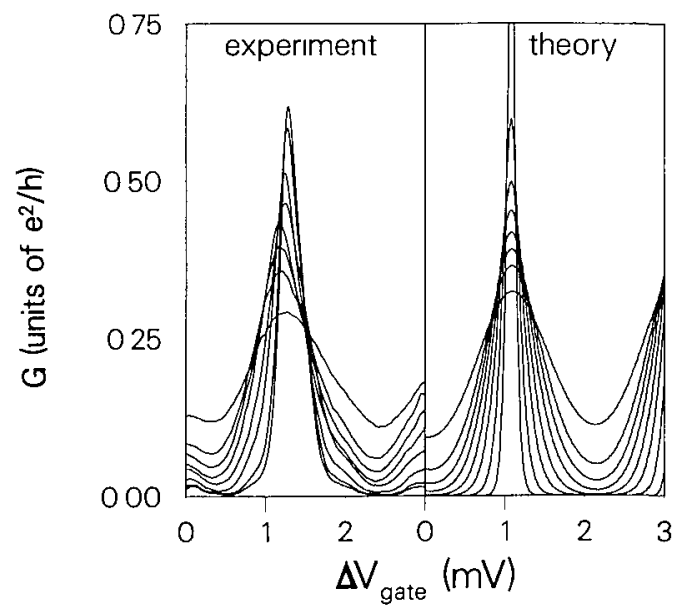

FIG. 8. Left panel: single conductance peak of channel $D 1$ at $B=6.66 \mathrm{~T}$. The temperatures are $110,190,290,380$, $490,590,710$, and $950 \mathrm{mK}$, from highest to lowest peak. Right panel: line shape calculated from Eq. (9) for $e^{2} / C=0.53$ $\mathrm{meV}, \Delta E=0.044 \mathrm{meV}, \alpha=0.265$, and $h \Gamma^{l, r}=0.065 \mathrm{meV}$.

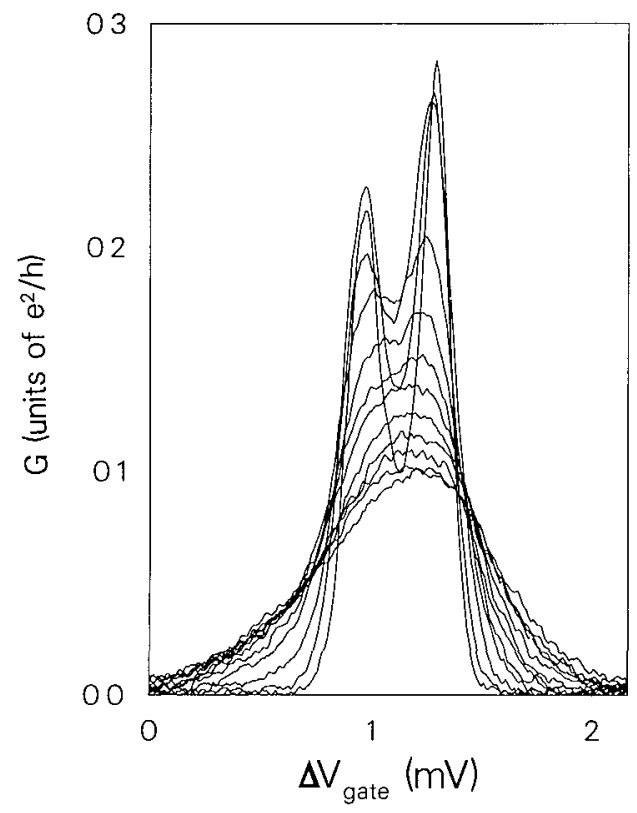

FIG. 9. Single conductance peak of channel $D 1$ at $B=$ $6.66 \mathrm{~T}$. The temperatures are $65,140,195,245,350,485,680$, and $845 \mathrm{mK}$, from highest to lowest peak.

at $2 e^{2} / h$ is no longer visible. Instead, there is some evidence of a Hall plateau at $G=e^{2} / h$. In addition, there is a plateaulike feature near $G=\frac{1}{2} e^{2} / h$, reminiscent of that reported by Timp et al. ${ }^{26}$ in a four-terminal measurement. Finally, we note that in the regime where the dips occur, the conductance at $1.5 \mathrm{~K}$ is below the average conductance at $50 \mathrm{mK}$, while in the regime of the periodic conductance peaks at more negative gate voltages the ordering is reversed. As discussed in Sec. V, the dips in the conductance at $50 \mathrm{mK}$ can be explained by resonant reflection in the channel.

The left panel of Fig. 8 shows the temperature dependence of one of the peaks in the conductance of channel $D 1$ at $B=6.66 \mathrm{~T}$. $\Lambda \mathrm{t}$ the lowest temperatures, this was one of the most pronounced peaks present in the conductance trace as a function of gate voltage. The

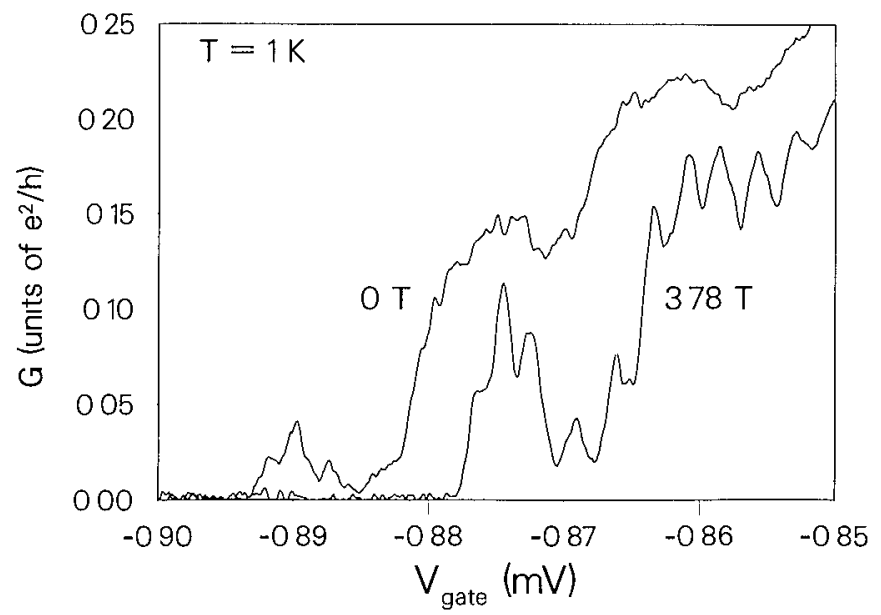

FIG. 10. Two-terminal conductance vs gate voltage of channel $U 2$ at $T=1 \mathrm{~K}$, and $B=0$ and $3.78 \mathrm{~T}$. 
peak height increases with decreasing temperature, and reaches a value of $0.6 e^{2} / h$ at $T=100 \mathrm{mK}$. Note the opposite temperature dependence for channel $U 3$ at $B=0$ given in Fig. 4. As discussed in Sec. IV B, the reason for this difference is that the latter data are in the high-temperature classical regime where $k_{B} T$ exceeds the average level spacing $\Delta E$ of the conductance-limiting segment, whereas the data in Fig. 8 are in the lowtemperature quantum regime $k_{B} T \lesssim \Delta E$. The calculated traces in Fig. 8, right panel, are discussed in Sec. IV B.

We often find fine structure developing on the conductance peaks. An example of this behavior is shown in Fig. 9, for another peak in the conductance of channel $D 1$, at $B=6.66 \mathrm{~T}$. For temperatures below $250 \mathrm{mK}$, the peak is split into a doublet. The amplitudes of both parts increase with decreasing temperature, and become better resolved as well, due to a reduction in width. We find that conductance peaks which show such fine structure typically are smaller than those that do not (note the difference in vertical scale in Figs. 8 and 9). As discussed in Sec. IV C, this can be understood from the presence of multiple segments in the wire.

The conductance oscillations in the samples without intentional Be doping are enhanced by a magnetic field similar to those observed in the Be-doped samples. We give one example, in Fig. 10, for channel $U 2$ at $T=1$ $\mathrm{K}$. Only the trace at $B=3.78 \mathrm{~T}$ shows rapid periodic oscillations.

\section{Magnetoconductance fluctuations}

Whereas the conductance as a function of gate voltage at fixed magnetic field shows periodic oscillations, no such behavior is observed when the magnetic field is varied and the gate voltage is fixed. As shown in Fig. 11, the duality between variations in the gate voltage and magnetic field, applicable to the quantum ballistic, adiabatic, and diffusive transport regimes ${ }^{15}$ breaks down in our samples. We have studied the four-terminal longitudinal magnetoconductance $G_{L}$, using sample $D 3$, which has the miniature Hall-bar geometry shown in the inset of Fig. 11(b) [see also Fig. 1(b)]. As shown in Fig. 11(a), the four-terminal magnetoconductance at $T=50 \mathrm{mK}$ exhibits essentially random structure, whereas in Fig. 11(b) it can be seen that the conductance as a function of gate voltage for the same sample exhibits periodic oscillations. [The two-terminal magnetoconductance has no periodic oscillations as a function of the magnetic field either (not shown).] The extreme sensitivity of the magnetoconductance to a small change in the gate voltage is not surprising, since the measurements were made for gate voltages in the regime where the conductance oscillates periodically as a function of $V_{\text {gate }}$ [at least for the top two panels in Fig. 11(a), cf. Fig. 11(b)]. As we will discuss in Sec. V, we interpret the absence of periodic magnetoconductance oscillations as a manifestation of the Coulomb blockade of the Aharonov-Bohm effect.

The magnetoconductance trace shown in the bottom panel of Fig. 11(a) (note the difference in vertical scale) was obtained at a gate vollage just outside the regime of periodic conductance oscillations. The large peaks in the conductance near 2.5 and $6 \mathrm{~T}$ in this trace are resistance minima, reminiscent of Shubnikov-de Haas oscillations in the quantum Hall effect regime. The lattcr can be identified quite well as the channel width is increased further, in which case the resistance at the minima approaches zero, and $G_{L}$ acquires very large values. From a set of measurements of the Shubnikov-de Haas oscillations at several values of the gate voltage, we found by extrapolation a value of $n_{s} \sim 1.5 \times 10^{11} \mathrm{~cm}^{-2}$ for the density in the channel in the regime of periodic conductance oscillations.

\section{Hall resistance}

The Hall resistance can be measured within the narrow channel using the miniature Hall-bar geometry of Fig. 1. The results for sample $D 3$ are shown in Fig. 12, for the same set of gate voltages as in Fig. 11. We find no qualitative differences in traces of the Hall resistance versus
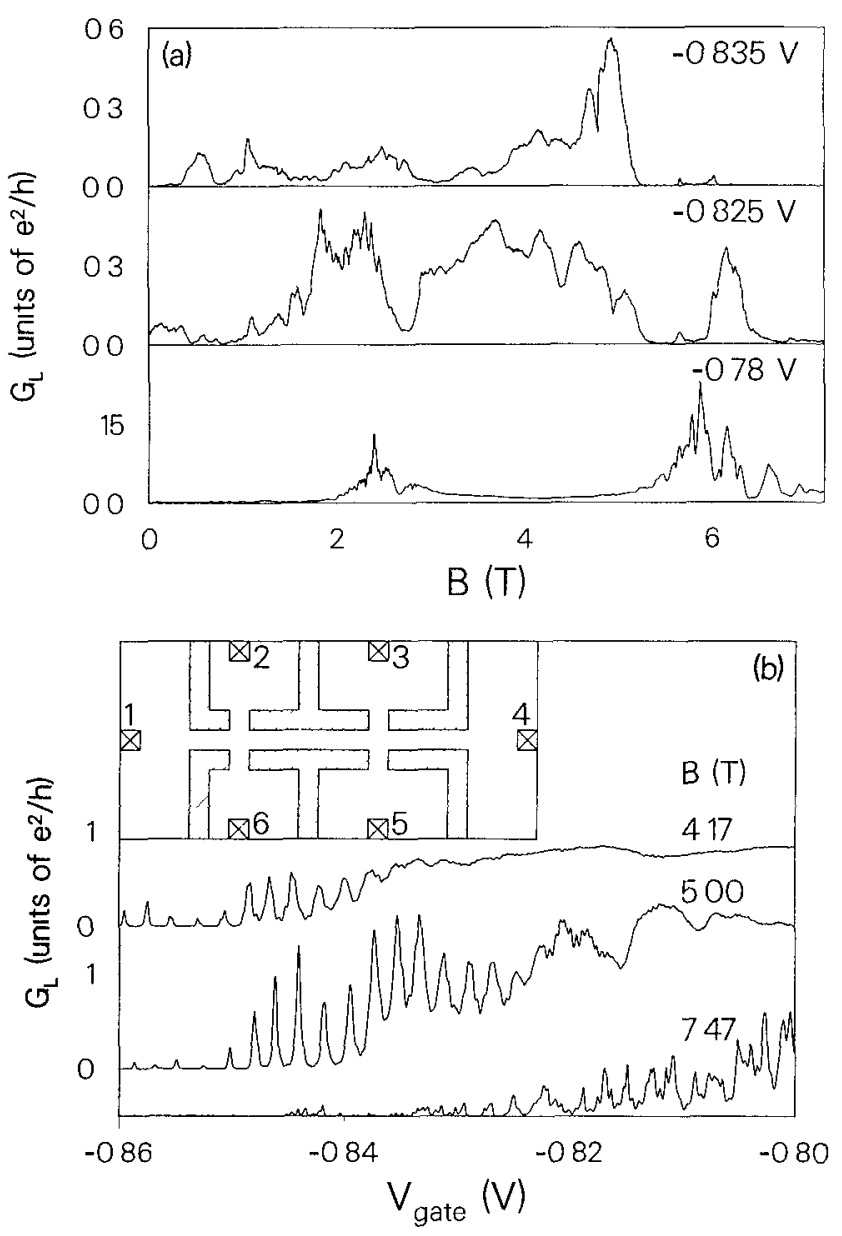

FIG. 11. (a) Four-terminal longitudinal conductance $G_{L}$ of channel $D 3$ at $T=50 \mathrm{mK}$ as a function of magnetic field, for three values of the gate voltage. (b) $G_{L}$ as a function of gate voltage for channel $D 3$ at $T=50 \mathrm{mK}$, for three values of the magnetic field. Inset: schematic top view of the miniature Hall-bar geometry. Contacts 1 and 4 were used as current contacts, and the voltage was measured across contacts 2 and 3 . 


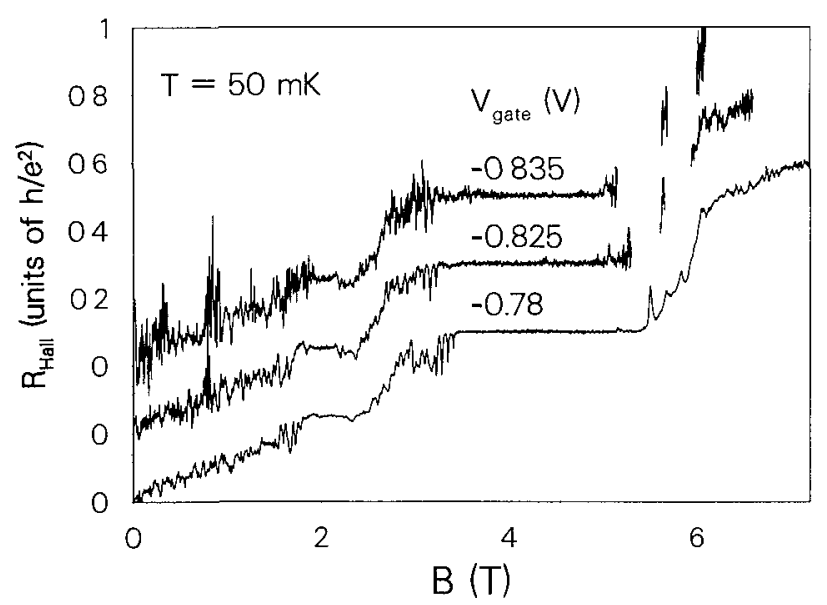

FIG. 12. Hall resistance of channel $D 3$ at $\mathrm{T}=50 \mathrm{mK}$, for three values of the gate voltage. The Hall resistance cannot be measured when the conductance of the channel is reduced to zero, hence the interruptions in the traces around $6 \mathrm{~T}$. The small channel conductance is also responsible for the poor signal-to-noise ratio of these experimental traces. Contacts 1 and 4 were used as current contacts, and the Hall voltage was measured across contacts 3 and 5 [see inset in Fig. 11(b)].

magnetic field in the regime of periodic conductance oscillations and traces obtained outside this regime. The Hall resistance cannot be measured in ranges of the magnetic field where the conductance is close to zero (cf. Fig. 11). This is the reason for the missing parts in the traces at $V_{\text {gate }}=-0.825$ and $-0.835 \mathrm{~V}$ in Fig. 12 .

In all traces in Fig. 12, the quantum Hall plateau at $2 e^{2} / h$ can be recognized easily, but the plateau at $4 e^{2} / h$ is less pronounced. (The spin-split plateaus at odd multiples of $e^{2} / h$ are not resolved in the narrow channels.) In between the plateaus, quasiperiodic oscillations as a function of magnetic field are found (see, for example, near 3 $\mathrm{T}$ in the trace at $\left.V_{\text {gate }}=-0.78 \mathrm{~V}\right)$. We attribute these to an Aharonov-Bohm effect involving resonant reflection. (The Coulomb blockade of the Aharonov-Bohm effect mentioned in Sec. IIIC refers to the two-terminal conductance, not to the Hall resistance.) Below $2 \mathrm{~T}$ the Hall resistance shows random oscillations. For $V_{\text {gate }}=-0.825$ and $-0.835 \mathrm{~V}$, these are time dependent and not reproducible (the signal-to-noise ratio in this regime is poor, because of the low conductance of the narrow channel). To the extent that the fluctuations are reproducible, we attribute these to quantum interference effects familiar from other studies of narrow channels. ${ }^{27}$

We also have tried to measure the IIall resistance (at fixed magnetic field) as a function of gate voltage. In the regime of periodic conductance oscillations this is very difficult for the same reason mentioned above: The Hall resistance cannot be measured when the two-terminal conductance is reduced to zero. It therefore cannot be established experimentally whether periodic oscillations occur in the Hall resistance. One could argue that this question is meaningless.

\section{COULOMB-BLOCKADE OSCILLATIONS}

In this section we analyze those features of our experimental results that may be considered to be generic, rather than sample specific. The most conspicuous are the conductance oscillations periodic in the gate voltage. The value of the period, its insensitivity to a strong magnetic field, the absence of spin-splitting, and the absence of magnetoconductance oscillations, can all be understood on the basis of a general formula ${ }^{6}$ expressing the condition for a conductance peak at $T=0$, see Sec. IV A. The temperature dependence of the amplitude and width of the oscillations is analyzed in terms of a kinetic theory for the conductance of a quantum dot in the regime of comparable charging energy and level spacing. ${ }^{7}$ This is the subject of Sec. IV B. In these two subsections we assume that the Coulomb-blockade oscillations arise from a single conductance-limiting segment. In Sec. IV C we briefly consider the effects of multiple segments in series.

\section{A. Periodicity}

We model the conductance-limiting segment in the narrow channel as a quantum dot, which is weakly coupled by tunnel barriers to two leads [see Fig. 13(a)]. The dot contains a set of energy levels $E_{p}$, measured relative to the bottom of the potential well in the dot. In the absence of charging effects, a conductance peak due to resonant tunneling occurs when the Fermi level $E_{F}$ in the leads lines up with one of the levels in the dot. To determine the location of the conductance peaks as

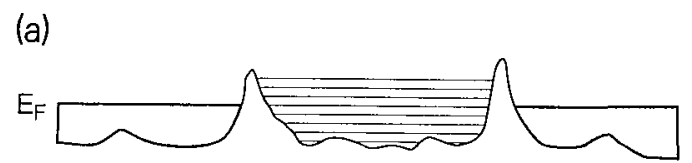

(b)

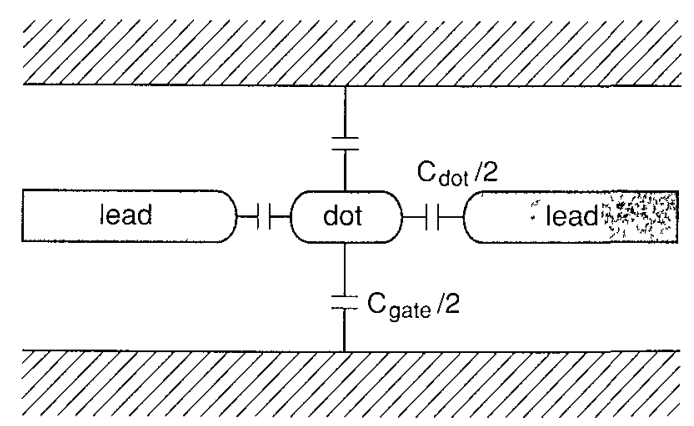

FIG. 13. (a) Schematic conductance band diagram of a disordered quantum wire containing a conductance-limiting segment (a quantum dot with a discrete energy spectrum). The leads are thought to have a continuous energy spectrum. (b) Equivalent circuit of quantum wire and split gate. The mutual capacitance of leads and gate is much larger than that of dot and gate $\left(C_{\text {gate }}\right)$, or dot and leads $\left(C_{\mathrm{dot}}\right)$, and can be neglected. 
a function of gate voltage requires only consideration of the equilibrium properties of the system. ${ }^{6}$ The condition for the $N$ th conductance peak is

$$
E_{N}^{*} \equiv E_{N}+\left(N-\frac{1}{2}\right) \frac{e^{2}}{C}=E_{F}+e \phi_{\mathrm{ext}} .
$$

Here $C$ is the capacitance of the dot, and $\phi_{\text {ext }}$ represents the electrostatic potential difference of the dot and leads due to external charges (see below). The left-hand side of Eq. (1) defines a renormalized energy level $E_{N}^{*}$. The average renormalized level spacing $\Delta E^{*}=\Delta E+e^{2} / C$ is enhanced above the average bare level spacing $\Delta E$ by the charging energy. In the limit $e^{2} / C \ll \Delta E$, Eq. (1) is the usual condition for resonant tunneling of noninteracting electrons. In the limit $e^{2} / C \gg \Delta E$, Eq. (1) is the condition for a peak for classical Coulomb-blockade oscillations in the conductance. ${ }^{28-32}$

Experimentally, we study the Coulomb-blockade oscillations as a function of gate voltage rather than Fermi energy $E_{F}$. To determine the periodicity from Eq. (1), we need to know how $E_{F}$ and the set of levels $E_{p}$ depend on $\phi_{\text {ext }}$. The external charges determining $\phi_{\text {ext }}$ are supplied by the ionized donors in the doped $\mathrm{Al}_{x} \mathrm{Ga}_{1-x}$ As layer and by the gate electrodes (with an electrostatic potential difference $\phi_{\text {gate }}$ between gates and 2DEG). We have

$$
\phi_{\mathrm{ext}}=\phi_{\mathrm{donors}}+\alpha \phi_{\mathrm{gatc}}
$$

where $\alpha$ (as well as $C$ ) is a rational function of the capacitance matrix elements of the system. For split-gate quantum wires it is reasonable to assume that on average the electron gas densities in the dot and leads increase equally fast with $\phi_{\text {gate, }}$ both being affected equally by the gates. In that case $E_{F}-E_{N}$ has approximately the same value at each conductance peak. The period of the oscillations now follows from Eqs. (1) and (2),

$$
\Delta \phi_{\text {gate }}=\frac{e}{\alpha C} .
$$

To clarify the meaning of the parameters $C$ and $\alpha$, we represent the system of the dot, gates, and leads by the equivalent circuit of Fig. 13(b). The mutual capacitance of gates and leads does not enter our problem explicitly, since it is much larger than the mutual capacitances of the gate and $\operatorname{dot}\left(C_{\text {gate }}\right)$ and the dot and leads $\left(C_{\text {dot }}\right)$. The capacitance $C$ determining the charging energy $(N-$ $\left.\frac{1}{2}\right) e^{2} / C$ is formed by $C_{\text {gate }}$ and $C_{\text {dot }}$ in parallel,

$$
C=C_{\text {gate }}+C_{\text {dot }} .
$$

The period of the oscillations corresponds to the increment by $e$ of the charge on the dot with no change in the voltage across $C_{\text {dot }}$. This implies $\Delta \phi_{\text {gate }}=e / C_{\text {gate }}$, or

$$
\alpha=\frac{C_{\text {gate }}}{C_{\text {gate }}+C_{\text {dot }}}
$$

Thus, in terms of the electrostatic potential difference between gate and leads, the period of the conductance oscillations is $\Delta \phi_{\text {gate }}=e / C_{\text {gate }}$. Note that this result applies regardless of the relative magnitudes of $\Delta E$ and $e^{2} / C$.

The experimental gate voltage is the electrochemical potential difference $V_{\text {gate }}$ between gate and leads, i.e., the difference in Fermi levels, rather than the electrostatic potential-difference $\phi_{\text {gate }}$, i.e., the difference in conduction-band bottoms. In one period, the change in Fermi energy in the dot and leads (measured relative to the local conduction-band bottom) is approximately equal to $\Delta E$. The change in Fermi energy in the (metal) gate is negligible, because the density of states in a metal is much larger than in a $2 D E G$. We thus find for the oscillation period in terms of the electrochemical potential difference

$$
\Delta V_{\text {gate }}=\frac{\Delta E}{e}+\Delta \phi_{\text {gate }}=\frac{\Delta E}{e}+\frac{e}{C_{\text {gate }}} .
$$

Note that $C_{\text {dot }}$ does not affect the periodicity.

In the case of a twofold spin degeneracy, the level spacing $E_{p+1}-E_{p}$ in the dot alternates between 0 and $\Delta E$, where $\Delta E$ is the spacing of the degenerate levels. This leads to a doublet structure in the oscillations as a function of $E_{F}$. To determine the peak spacing as a function of gate voltage we approximate the change in $E_{F}$, with $\phi_{\text {gate }}$ by $\partial E_{F} / \partial \phi_{\text {gate }} \sim C_{\text {gate }} \Delta E / 2 e$. We then obtain from Eqs. (1), (2), (4), and (5) that the spacing alternates between two values:

$$
\begin{aligned}
\Delta \phi_{\text {gate }}^{(1)} & =\frac{e}{C_{\text {gate }}} \frac{e^{2} / C}{\frac{1}{2} \Delta E+e^{2} / C}, \\
\Delta \phi_{\text {gate }}^{(2)} & =\frac{e}{C_{\text {gate }}} \frac{\Delta E+e^{2} / C}{\frac{1}{2} \Delta E+e^{2} / C} .
\end{aligned}
$$

The average spacing equals $e / C_{\text {gate, }}$ in agreement with Eq. (3) (derived for nondegenerate levels). To obtain $\Delta V_{\text {gate }}$ one has to replace the term $e / C_{\text {gate }}$ in Eqs. (7) and (8) by $e / C_{\text {gate }}+\Delta E / 2 e$. If the charging energy dominates $\left(e^{2} / C \gg \Delta E\right)$ one has equal spacing $\Delta \phi_{\text {gate }}^{(1)}=\Delta \phi_{\text {gate }}^{(2)}=e / C_{\text {gate }}$, as for nondegenerate levels. In the opposite limit $\Delta E \gg e^{2} / C$, one finds $\Delta \phi_{\text {gate }}^{(1)}=0$, and $\Delta \phi_{\text {gate }}^{(2)}=2 e / C_{\text {gate }}$ instead. Thus, the period is effectively doubled, corresponding to the addition of two electrons to the dot, instead of one. This is characteristic for resonant tunneling of noninteracting electrons through spin-degenerate energy levels. An external magnetic field resolves the spin degeneracy in this case, leading to a splitting of the conductance peaks which increases with the field. This is not observed in our experiments.

We now apply these results to our experimental situation. We recall that no correlation is found between the periodicity of the oscillations and the channel length, and that the conductance oscillations are observed when the width is reduced below $W \sim 0.1 \mu \mathrm{m}$, in which case the electron density is $1.5 \times 10^{11} \mathrm{~cm}^{-2}$. A $3-\mu \mathrm{m}$-long channel then contains some 450 electrons.

To calculate $C_{\text {dot }}$ and $C_{\text {gate }}$ is a rather complicated three-dimensional electrostatic problem, hampered further by the uncertain dimensions of the conductancelimiting segment. Experimentally, a typical spacing of the conductance peaks is $\Delta V_{\text {gate }} \sim 2.3 \mathrm{mV}$, so that from 
Eq. (6) we estimate $C_{\text {gate }} \sim 0.7 \times 10^{-16} \mathbf{F}$, ignoring the contribution of the finite level spacing to the period in gate voltage ( $\Delta E$ is typically much smaller than $e^{2} / C_{\text {gate }}$, see below). The length $L$ of the segment may be estimated from the gate voltage range between channel definition and pinch-off, $\delta V_{\text {gate }} \sim e n_{s} W_{\text {lth }} L / C_{\text {gate }}$, where $n_{s}=2.9 \times 10^{11} \mathrm{~cm}^{-2}$ is the electron density in the channel at definition. From the above estimate of $C_{\text {gate }}$ and using $\delta V_{\text {gate }} \sim 1 \mathrm{~V}$, we estimate $L \sim 0.3 \mu \mathrm{m}$. The resulting value for the capacitance per unit length $C_{\text {gate }} / L$ is consistent with what one would expect for the mutual capacitance per unit length of a wire of diameter $W$ running in the middle of a gap of width $W_{\mathrm{lth}}$ in a metallic plane ${ }^{33}$ (the thickness of the $\mathrm{Al}_{x} \mathrm{Ga}_{1-x}$ As layer between the gate and the 2DEG is small compared to $\left.W_{\text {lith }}\right): C_{\text {gate }} / L \sim 4 \pi \epsilon / 2 \operatorname{arccosh}\left(W_{\text {lth }} / W\right) \sim 3 \times 10^{-10}$ $\mathrm{F} / \mathrm{m}$.

The level spacing in the segment is estimated at $\Delta E \sim$ $2 \pi \hbar^{2} / \mathrm{mLW} \sim 0.2 \mathrm{meV}$ (for a twofold spin degeneracy). Since each oscillation corresponds to the addition of a single electron to the dot, the maximum number of oscillations following from $\Delta E$ and the Fermi energy $E_{F} \sim 5 \mathrm{meV}$ when the dot is formed is given by $2 E_{F} / \Delta E \sim 50$, consistent with the observations. From the fact that the oscillations are still observable at $T=1.5 \mathrm{~K}$, albeit with considerable thermal smearing, we deduce that in our experiments $e^{2} / C+\Delta E \sim 1 \mathrm{meV}$. Thus, $C \sim 2 \times 10^{-16} \mathrm{~F}, C_{\text {dot }}=C-C_{\text {gate }} \sim 1.3 \times 10^{-16}$ $\mathbf{F}$, and $\alpha \equiv C_{\text {gate }} / C \sim 0.35$. The mutual capacitance of dot and leads $\left(C_{\text {dot }}\right)$ may be approximated by the sclfcapacitance of the dot, which should be comparable to that of a two-dimensional circular disk ${ }^{33}$ of diameter $L$ (which is the largest linear dimension of the elongated conductance-limiting segment), $C_{\text {dot }} \sim 4 \epsilon L \sim 1.4 \times 10^{-16}$ F, consistent with the above estimatc.

We conclude that the periodicity of the conductance oscillations in our experiment is explained consistently by the theory for Coulomb-blockade oscillations, in a regime where $e^{2} / C$ is larger than the bare level-spacing $\Delta E$ by about a factor of 4 . According to Eq. (6), the period is governed by $e / C_{\text {gate }}$, which exceeds $\Delta E / e$ by an order of magnitude, thus providing part of the explanation of the regularity of the oscillations. A finite temperature $k_{B} T>$ $\Delta E$ further regulates the spacing of the oscillations, see Sec. IV C.

As an alternative explanation of the conductance oscillations, resonant tunneling of noninteracting electrons has been proposed. ${ }^{12,13}$ As mentioned in the Introduction, there are several compelling arguments for rejecting this explanation. Firstly, ${ }^{14}$ the measured activation energy of the conductance minima would imply a bare level spacing $\Delta E \sim 1 \mathrm{meV}$ if charging effects would be absent. Since the Fermi energy $E_{F}$ is $5 \mathrm{meV}$ or less, such a large level spacing would restrict the possible total number of oscillations in a gate voltage scan to a maximum of $2 E_{F} / \Delta E \sim 10$, considerably less than the number observed experimentally. ${ }^{1,14}$ Secondly, one would expect a spin splitting of the oscillations in a strong magnetic field, which is not observed. ${ }^{9}$ Finally, the fact that no oscillations are found as a function of magnetic field ${ }^{9,14}$ all but rules out resonant tunneling of noninteracting electrons as an explanation of the oscillations as a function of gate voltage.

\section{B. Amplitude and line shape}

Equation (1) is sufficient to determine the periodicity of the conductance oscillations but not their amplitude and width, which requires the solution of a kinetic equation. The nonlinear response regime has been studied by Averin, Korotkov, and Likharev ${ }^{34}$ The linear response solution of present interest was obtained by Beenakker, ${ }^{7}$ and generalizes earlier results by Kulik and Shekhter ${ }^{29}$ in the classical regime. Results equivalent to Ref. 7 have been obtained independently by Meir, Wingreen, and Lee, ${ }^{8}$ by a different method. In this subsection we give the general formula for the conductance and summarize the underlying assumptions. Using this formula, we calculate the conductance for our experimental conditions, and compare it to our data.

Reference 7 applies to a quantum dot which is weakly coupled by tunnel barricrs to two electron gas reservoirs. A continuum of states is assumed in the reservoirs. The tunnel rates from level $p$ in the quantum dot to the left and right reservoirs are denoted by $\Gamma_{p}^{l}$ and $\Gamma_{p}^{r}$, respectively. It is assumed that, near the Fermi energy in the quantum dot, both the level spacing $\Delta E$ and the thermal energy $k_{B} T$ are much greater than the intrinsic width of the encrgy levels $h \Gamma \equiv h\left(\Gamma^{l}+\Gamma^{r}\right)$. This assumption allows a characterization of the state of the quantum dot by a set of occupation numbers, one for each energy level. It is assumed also that inelastic scattering takes place exclusively in the reservoirs, not in the quantuin dot. (The effects of inelastic scattering in the dot are discussed in Ref. 7.)

By solving the kinetic equation in linear response, it is found that

$$
\begin{aligned}
& G=\frac{e^{2}}{k_{B} T} \sum_{p=1}^{\infty} \sum_{N=1}^{\infty} \frac{\Gamma_{p}^{l} \Gamma_{p}^{r}}{\Gamma_{p}^{l}+\Gamma_{p}^{r}} P_{\mathrm{eq}}\left(N, n_{p}=1\right) \\
& \times\left\{1-f\left[E_{p}+U(N)-U(N-1)\right.\right. \\
&\left.\left.-E_{F}\right]\right\} .
\end{aligned}
$$

Here $P_{\text {eq }}\left(N, n_{p}=1\right)$ is the joint probability that the quantum dot contains $N$ electrons and that level $p$ is occupied (see the Appendix), $f(x) \equiv\left[1+\exp \left(x / k_{B} T\right)\right]^{-1}$ is the Fermi-Dirac distribution function, and $U(N)=$ $(\mathrm{Ne})^{2} / 2 \mathrm{C}-\mathrm{Ne} \phi_{\mathrm{ext}}$ is the charging energy. The product of distribution functions expresses the fact that tunneling of an electron from an initial state $p$ in the dot to a final state in the reservoir requires an occupied initial state and an empty final state.

Limiting cases of Eq. (9) are discussed in Ref. 7 (see also Ref. 23). The conductance $G_{\min }$ in the minima of the oscillations depends exponentially on the temperature, $G_{\mathrm{mm}} \propto \exp \left(-E_{\mathrm{act}} / k_{B} T\right)$, with activation energy ${ }^{7}$

$$
E_{\text {act }}=\frac{1}{2}\left(\Delta E+e^{2} / C\right)=\frac{1}{2} \Delta E^{*} .
$$

This result holds for equal tunncl rates to two subsequent energy levels. The renormalized level spacing $\Delta E^{*} \equiv \Delta E+e^{2} / C$, thus equals twice the activation 
energy of the conductance minima. The exponential decay of the conductance at the minima of the Coulombblockade oscillations results from the suppression of tunneling processes which conserve energy in the intermediate state in the quantum dot. Tunneling via a virtual intermediate state is not suppressed at low temperatures, and becomes important when $k_{B} T \lesssim h \Gamma{ }^{35,36} \mathrm{In}$ the opposite case these virtual tunnel processes can be neglected.

In Fig. 4 we compare a calculation based on Eq. (9) with experimental traces for channel $D 1$, discussed in Sec. III A. To obtain good agreement we assume that the tunnel rates for successive spin-degenerate levels increase linearly as $\Gamma_{i}^{l}=\Gamma_{i}^{r}=0.027 i \Delta E / h$, where $\Delta E=0.1$ $\mathrm{meV}$ is the spacing of these levels. Both the increase of the tunnel rates with energy and the low number of electrons assumed to be present in the dot are necessary for obtaining a good agreement with the experiment. (In the calculation, the first conductance peak corresponds to an occupation of the dot by zero or one electron.) The capacitances were chosen so that $e^{2} / C=0.6 \mathrm{meV}$ and $\alpha=0.265$. These values are consistent with the estimates given above. The Fermi energy in the leads was assumed to increase with gate voltage such that it is on average equal to the energy of the highest occupied level in the dot at $T=0$ (cf. Sec. IV A). The data in Fig. 4 are in the classical regime $\left(k_{B} T>\Delta E\right)$, where the peak height is roughly independent of temperature, whereas the width of the peaks increases with $T$. This is reproduced by our calculations.

On lowering the temperature, we enter the resonant tunneling regime $k_{B} T<\Delta E$. As long as $k_{B} T>h \Gamma$, the width of the peaks is proportional to $T$ and the peak height is proportional to $1 / T$. The peak height thus increases on lowering the temperature, up to a value of order $e^{2} / h$, reached when $k_{B} T$ is of order $h \Gamma$. A theory for the regime $k_{B} T<h \Gamma$ is not available presently, but we surmise that the maximum peak height is $e^{2} / h$, for the case of equal tunnel barriers. This is consistent with our experimental observations, which do not show conductance peaks exceeding this value. [The largest conductance peaks found experimentally approach $e^{2} / h$, see Fig. 6(c) (channel $D 1$, at $5 \mathrm{~T}$ ).]

To test to what extent Eq. (9) can describe our experimental results in the quantum regime $k_{B} T \lesssim \Delta E$, we have calculated the peaks shown in the right panel of Fig. 8. (The data in the left panel of Fig. 8 was obtained in the presence of a magnetic field of $6.66 \mathrm{~T}$, so that we assume no spin degeneracy in the calculation.) Equation (9) reproduces the temperature dependence of the peak height and width quite well, for temperatures between 190 and $950 \mathrm{mK}$. The parameter values used are $e^{2} / C=0.53 \mathrm{meV}, \Delta E=0.088 \mathrm{meV}, \alpha=0.265$, and $h \Gamma^{l}=h \Gamma^{r}=0.065 \mathrm{meV}$, which are consistent with the values used for the calculations shown in the bottom panel of Fig. 4. The Zeeman spin-splitting energy is not known, due to uncertainties in the $g$ factor, but is taken equal to $\frac{1}{2} \Delta E$ in the calculations. The resulting set of equidistant nondegenerate levels is spaced at $0.044 \mathrm{meV}$. We note, however, that the parameter values used imply that $k_{B} T<h \Gamma$ for the calculated peaks in Fig. 4, so that
Eq. (9) is strictly not valid, and instead a theory should be used which takes the finite broadening of the levels in the quantum dot into account.

The data obtained in the absence of a magnetic field at very low temperatures [see Figs. 2 and 6(c)] is probably in the quantum regime as well. An analysis of these data is hampered by the presence of multiple segments in the wire, as discussed in Sec. IV C. A strong magnetic field reduces the backscattering probability in the channel, which may explain why the conductance at low $T$ is less affected by their presence. The agreement between theory and experiment in Figs. 4 and 8, for a reasonably consistent set of parameter values, and over a wide range of temperatures, supports our interpretation of the conductance oscillations periodic in the gate voltage as Coulomb-blockade oscillations in the regime of comparable level spacing and charging energy.

\section{Multiple segments}

In an attempt to investigate the effects of multiple segments in the wire, we consider the conductance of two decoupled quantum dots of different size in series. This simple model can illustrate some aspects of the experimental data. Among these are the observation of regular oscillations at relatively high temperatures, which are replaced by irregularly spaced peaks at millikelvin temperatures, and the splitting exhibited by some of the regular peaks on decreasing the temperature.

The calculations proceed as follows: Using Eq. (9) we calculate the conductances $G_{1}$ and $G_{2}$ of the two dots individually. The resulting conductance of the dots in series is obtained via Ohmic addition $\left(G^{-1}=G_{1}^{-1}+G_{2}^{-1}\right)$, i.e., it is assumed that the dots are separated by a reservoir. The parameter values for the first dot were chosen equal to those used to model the peak in Fig. 8: $e^{2} / C_{1}=0.53 \mathrm{meV}$ and $\alpha_{1}=0.265$, but with twofolddegenerate levels, randomly spaced within a bandwidth of $25 \%$ around the average spacing $\Delta E_{1}=0.088 \mathrm{meV}$. The tunnel rates were chosen to vary randomly within a bandwidth of $50 \%$ around the average tunnel rates $h \Gamma^{l}=h \Gamma^{r}=0.065 \mathrm{meV}$. The parameter values for the second dot were obtained using a scaling argument. It is assumed that the relevant capacitances $C$ and $C_{\text {gate }}$ are approximately proportional to the length $L$ of the conductance-limiting segment (see Sec. IV A), while the average level spacing $\Delta E \propto 1 / L$ and the parameter $\alpha$ is independent of $L$. The second dot was chosen to be approximately 2.7 times as long as the first dot, and accordingly we have used $e^{2} / C_{2}=0.097 \mathrm{meV}, \alpha_{2}=0.273$, $\Delta E_{2}=0.033 \mathrm{meV}$, and $h \Gamma^{l}=h \Gamma^{r}=0.065 \mathrm{meV}$ (the energy levels and tunnel rates were chosen randomly within the same bandwidths as for the first dot). The results of the calculations are shown in Fig. 14.

Figure 14 illustrates several points. At the relatively high temperature of $1.5 \mathrm{~K}$, the conductance oscillations are very regular. The reason is that at this temperature the oscillations of the second dot are smeared completely, because $e^{2} / C_{1}>k_{B} T>e^{2} / C_{2}$. Additionally, since $k_{B} T>\Delta E$ the period is determined by an average 


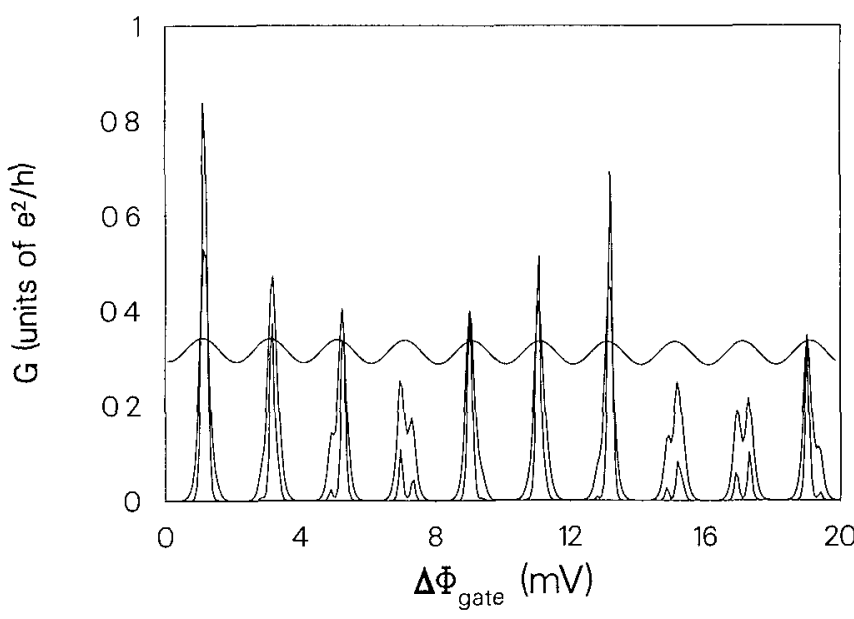

FIG. 14. Calculations of the conductance oscillations of two quantum dots in series, separated by a reservoir. The temperatures are $1.5 \mathrm{~K}, 240 \mathrm{mK}$, and $130 \mathrm{mK}$. The paramete values are given in the text.

lcvel spacing and tunnel rate, rather than by a particular level separation and tunnel rate for each individual peak. As the temperature is decreased, the quantum regime $k_{B} T<\Delta E$ is entered (in particular for the first dot), and the oscillations of the second dot become important since $k_{B} T<e^{2} / C_{2}$. The resulting irregularity in the conductance as a function of gate voltage is apparent from Fig. 14. In addition, it shows that at low temperatures a split of the peaks can result from differences in period and activation energy of the oscillations in the two dots. As in the experimental data, peaks exhibiting such a splitting are smaller than peaks that do not split. In contrast to the experimental data, however, the split peaks decrease rather than increase (sec Fig. 9) with decreasing temperature. This may be due to the the intrinsic broadening of the transmission resonances through the dot, which becomes important for $k_{B} T<h \Gamma$ and which is not accounted for by the calculations (cf. Sec. IV B).

An alternative model of a large and small quantum dot which are directly coupled (not via a reservoir, as in our calculation), has recently been studied by Glazman. ${ }^{37}$ They find a crossover from periodic Coulomb-blockade oscillations to aperiodic fluctuations at low temperatures, when $k_{B} T$ is smaller than the level spacing in both quantum dots. A conductance peak then requires that the levels in both the quantum dots line up, which occurs at random.

\section{DISCUSSION}

In this section we discuss those aspects of the data that are not so well understood, as well as the connection with other work. Our disordered quantum wires exhibit pertodzc conductance oscillations as a function of gate voltage. This effect has also been observed in electron and hole gases in Si (Refs. 1, 9, and 17) and in the electron gas in GaAs. ${ }^{14,16}$ In contrast, earlier work by Fowler and co-workers ${ }^{38}$ and by $K$ wasnick et al ${ }^{39}$ on narrow inver- sion and accumulation layers in $\mathrm{Si}$ has revealed sharp but aperadac conductance peaks. Structure reminiscent of their results is visible in some of our samples at low temperature $(50 \mathrm{mK})$, in zero or very strong magnetic fields [cf. Figs. 2 (lower left panel) and 6(c) (traces for $B=0$ and $7.59 \mathrm{~T}$ )]. How can these observations be reconciled? We surmise that the explanation is to be found in differences in strength and spatial scale of the potential fluctuations in the wires. Coulomb-blockade oscillations require two large potential spikes, which delimit a conductance-limiting segment in the quantum wire [Fig. 13(a)], containing a large number of states. The random conductance fluctuations seen previously ${ }^{38,39}$ are thought instead to be due to variable range hopping between individual localized states, distributed randomly along the length of the channel. ${ }^{40-42}$ As proposed by Glazman ${ }^{37}$ the periodic Coulomb-blockade oscillations of multiple segments in series can transform into sharp aperiodic fluctuations at low temperatures. This may explain our observation (Fig. 2) that periodic oscillations are found at temperatures around $1 \mathrm{~K}$, whereas irregular structure occurs at millikelvin temperatures. On increasing the Fermi energy, a transition to the diffusive transport regime occurs eventually, regardless of the type of disorder. Then both the Coulomb-blockade oscillations and the random conductance fluctuations due to variable range hopping are replaced by the "universal" conductance fluctuations characteristic of the diffusive transport regime. ${ }^{2,43,44}$

In very short channels $(0.5 \mu \mathrm{m}$ long and $1 \mu \mathrm{m}$ wide $)$ Fowler et $a l^{45}$ have found well-isolated, temperatureindependent (below $100 \mathrm{mK}$ ) conductance peaks, which they attributed to resonant tunneling. At very low temperatures a fine structure was observed, some of which was time dependent. A numerical simulation ${ }^{46}$ of the temporal fluctuations in the distribution of electrons among the available sites also showed fine structure if the time scale of the fluctuations is short compared to the measurement time, but large compared to the tunnel time. It is possible that a similar mechanism is responsible for some fine structure on the Coulomb-blockade oscillations in disordered quantum wires as well.

A curious phenomenon that we have found is the effect of a perpendicular magnetic field on the amplitude of the periodic conductance oscillations. The height of the conductance peaks is enhanced for intcrmediate field strengths $(1 \mathrm{~T} \lesssim B \lesssim 5 \mathrm{~T}$ ), but decreases again at stronger fields. The largest isolated peaks [found in channel $D 1$ at $5 \mathrm{~T}$, see Fig. 6(c)] approach a height of $e^{2} / h$, measured two terminally. A similar enhancement of the amplitude of the Coulomb-blockade oscillations by a magnetic field was observed in a quantum dot. ${ }^{20}$ One explanation is that the inelastic scattering rate is reduced by a magnetic field. In the low-temperature regime $k_{B} T \lesssim h \Gamma$ this presumably increases the peak height and decreases the width (see Ref. 7). In disordered quantum wires the magnetic suppression of backscattering provides another mechanism for an enhancement of the peak height because of the resulting reduced series resistance in the wire. Finally, the strong magnetic field regime in a wide high-mobility $2 \mathrm{DEG}$ is the realm of the fractional quantum Hall effect and the magnetic-field-induced tran- 
sition to the Wigner crystal. It is possible that the suppression of the Coulomb blockade oscillations for $B>6$ $\mathrm{T}$ is related in some unknown way to these phenomena.

For noninteracting electrons, one would expect to observe Aharonov-Bohm oscillations in the conductance of a quantum dot as a function of magnetic field in the quantum Hall effect regime. The reason is that such a dot has effectively a ring geometry if the magnetic length $l_{m} \equiv(\hbar / e B)^{1 / 2}$ is much smaller than the dot radius, due to the presence of circulating edge states. The AharonovBohm (AB) effect in such a dot may be interpreted as resonant tunneling through zero-dimenisional states. ${ }^{47,48}$ In the absence of Coulomb interaction, the period $\Delta B$ of the $\mathrm{AB}$ oscillations for a hard-wall $\operatorname{dot}$ of area $A$ is $\Delta B=h / e A$ (it may be larger for a soft-wall confining potential ${ }^{47}$ ). Such oscillations have indeed been observed in large quantum dots, ${ }^{47,49,50}$ but in our experiment at high magnetic fields, no periodic oscillations with the estimated $\Delta B \approx 0.1 \mathrm{~T}$ are found. Our observations are consistent with the Coulomb blockade of the AharonovBohm eflect. ${ }^{6}$ Each $\mathrm{AB}$ oscillation corresponds to an increase of the number of electrons in the dot by one. One can show from $\mathrm{Eq}$. (1) that the period of the $\Lambda \mathrm{B}$ oscillations is enhanced due to the charging energy, according to ${ }^{6}$

$$
\Delta B^{*}=\Delta B\left(1+\frac{e^{2}}{C \Delta E}\right)
$$

where $\Delta E$ is the energy level spacing of the circulating edge states. From our high-field expcriments we have estimated $e^{2} / C \Delta E \approx 10$ (cf. Fig. 8), so that $\Delta B^{*} \approx 10 \Delta B \approx 1 \mathrm{~T}$. The rapid $\mathrm{AB}$ oscillations in the magnetoconductance are therefore suppressed, notwithstanding the fact that oscillations can still be observed easily in a conductance trace as a function of gate voltage. The insensitivity of the period of the latter oscillations to a strong magnetic field is explained by the fact that the renormalized level spacing $\Delta E^{*} \approx e^{2} / C$ is approximately $B$ independent.

In one of our channels ( $D 1$, see Fig. 7 ) we have observed a crossover from resonant transmission at $G<$ $e^{2} / h$ (conductance peaks), to resonant reflection at $G>$ $e^{2} / h$ (conductance $d \imath p s$ ) at $T=50 \mathrm{mK}$. To explain the difference, we show schematically in Fig. 15 the boundaries of the quantum wire (thick lines), with the thin lines representing the edge channels which are formed in a strong magnetic field. ${ }^{15}$ Electrons can tunncl between the edge channels when they are close togcther, as indicated by the dashed lines. In Fig. 15(a) a conductance-limiting segment is formed because of the presence of two potential barriers or constrictions, and the conductance exhibits periodic Coulomb-blockade oscillations (Sec. IV). The temperature scale of these oscillations is set by the charging energy, which is relatively large. At less negative gate voltages, the guiding center energy of the edge channels near the Fermi level may exceed the height of the potential barriers. The edge channels are then transmitted adiabatically through the wire [Fig. 15(b)]. Backscattering can now occur due to tunneling between edge channels at opposite edges. This will happen predominantly near the potential barriers (dashed lines). The backscat- (a)

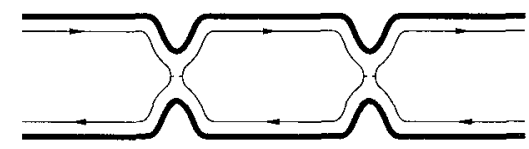

(b)

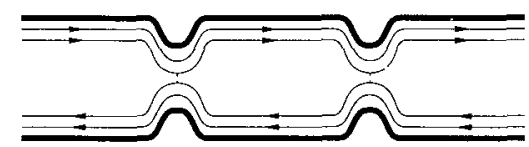

(c)

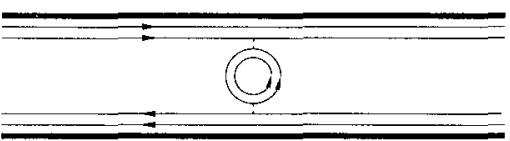

FIG. 15. Schematic view of the edge channels (thin lines) in the quantum wire, with a conductance-limiting segment (a), and without such a segment (b), (c).

tering can be enhanced resonantly due to constructive interference among these tumneling paths, leading to dips in the conductance. The strong temperature dependence of the conductance dips in Fig. 7 implies a low activation energy, indicating that charging effects do not affect the resonant backscattering significantly. Alternatively, resonant backscattering may occur also due to the presence of a circulating edge state in the center of the quantum wire, associated with a single potential spike. ${ }^{51}$ This mechanism is illustrated in Fig. 15(c). Experimentally we cannot discriminate between the two mechanisms.

In summary, we have reported on an experimental study of the periodic conductance oscillations as a function of gate voltage in split-gate disordered quantum wires in the 2DEG in a GaAs- $\mathrm{Al}_{x} \mathrm{Ga}_{1-x}$ As heterostructure. From the persistence up to a few kelvin of the dominant oscillations, and from the insensitivity of the period to a strong magnetic field, it is concluded that they are Coulomb-blockade oscillations. The appearance of additional periodicities and the onset of irregular conductance fluctuations at very low temperatures in some of the wires is attributed to the presence of multiple segments in these wires. We have compared the temperature dependence of the periodic conductance oscillations to a theory for Coulomb-blockade oscillations in the classical regime $k_{B} T>\Delta E$ and in the quantum regime $k_{B} T<\Delta E$. Good semiquantitative agreement with this theory is obtained, using physically reasonable parameter values, although our lowest temperature data appear to be in the intrinsically broadened resonance regime $k_{B} T<h \Gamma$, for which a theory has not yet been worked out. The effect of a perpendicular magnetic field on the oscillations is to enhance their amplitude at intermediate field strengths (between about $1 \mathrm{~T}$ and $5 \mathrm{~T}$ ), but to suppress them at stronger fields. This remains to be understood. In contrast to the traces of the conductance as a function of gate voltage, the magnetoconductance traces at constant gate voltage show no periodic oscillations. Since the conductance-limiting segment in our 
wires is essentially a quantum dot, we interpret this as experimental evidence for the Coulomb blockade of the Aharonov-Bohm effect.

\section{ACKNOWLEDGMENTS}

We thank M. A. A. Mabesoone and C. E. Timmering for technical support, B. W. Alphenaar, S. Colak, L. P. Kouwenhoven, L. W. Molenkamp, and J. G. Williamson for stimulating discussions, and M. F. H. Schuurmans and J. II. Wolter (Eindhoven University of Technology) for their support and interest. This research was partly funded by the ESPRIT basic research action Project No. 3133 .

\section{APPENDIX}

The joint probability $P_{\text {eq }}\left(N, n_{p}=1\right)$ appearing in Eq. (9) is defined in terms of the equilibrium distribution function of electrons among the energy levels, which is the Gibbs distribution in the grand canonical ensemble:

$$
\begin{array}{r}
P_{\text {eq }}\left(\left\{n_{i}\right\}\right)=Z^{-1} \exp \left[-\frac{1}{k_{B} T}\left(\sum_{i=1}^{\infty} E_{i} n_{i}+U(N)\right.\right. \\
\left.\left.-N E_{F}\right)\right], \quad
\end{array}
$$

where $\left\{n_{i}\right\} \equiv\left\{n_{1}, n_{2}, \ldots\right\}$ denotes a specific set of occupation numbers of the energy levels in the quantum dot. (The numbers $n_{i}$ can take on only the values 0 and 1.) The number of electrons in the $\operatorname{dot}$ is $N \equiv \sum_{i} n_{i}$, and $Z$ is the partition function,

$$
\begin{gathered}
Z=\sum_{\left\{n_{\mathbf{i}}\right\}} \exp \left[-\frac{1}{k_{B} T}\left(\sum_{i=1}^{\infty} E_{i} n_{i}+U(N)\right.\right. \\
\left.\left.-N E_{F}\right)\right]
\end{gathered}
$$

The joint probability $P_{\mathrm{eq}}\left(N, n_{p}=1\right)$ that the quantum dot contains $N$ electrons and that level $p$ is occupied is

$P_{\mathrm{eq}}\left(N, n_{p}=1\right)=\sum_{\left\{n_{\mathrm{\imath}}\right\}} P_{\mathrm{eq}}\left(\left\{n_{\imath}\right\}\right) \delta_{N, \sum_{\imath} n_{\mathrm{r}}} \delta_{n_{p}, 1}$
${ }^{1}$ J. H. F. Scott-Thomas, S. B. Field, M. A. Kastner, H. I. Smith, and D. A. Antoniadis, Phys. Rev. Lett. 62, 583 (1989).

2 J. C. Licini, D. J. Bishop, M. A. Kastner, and J. Melngailis, Phys. Rev. Lett. 55, 2987 (1985); W. J. Skocpol, P. M. Mankiewich, R. E. Iloward, L. D. Jackel, D. M. 'Tennant, and A. D. Stone, $\imath$ bid. 56, 2865 (1986).

${ }^{3}$ A. I. Larkin and P. A. Lee, Phys. Rev. B 17, 1596 (1978).

${ }^{4}$ P. A. Lee and T. M. Rice, Phys. Rev. B 19, 3970 (1979).

${ }^{5}$ H. van Houten and C. W. J. Beenakker, Phys. Rev. Lett. 63, 1893 (1989).

${ }^{6}$ C. W. J. Beenakker, H. van Houten, and A. A. M. Staring, Phys. Rev. B 44, 1657 (1991); in Granular Nanoelectronics, edited by D. K. Ferry (Plcnum, New York, 1991).

${ }^{7}$ C. W. J. Beenakker, Phys. Rev. B 44, 1646 (1991).

${ }^{8}$ Y. Meir, N. S. Wingreen, and P. A. Lee, Phys. Rev. Lett. 66, 3048 (1991).

${ }^{9}$ S. B. Field, M. A. Kastner, U. Meirav, J. H. F. ScottThomas, D. A. Antoniadis, H. I. Smith, and S. J. Wind, Phys. Rev. B 42, 3523 (1990).

${ }^{10}$ M. A. Kastner, S. B. Ficld, U. Meirav, J. H. F. ScottThomas, D. A. Antoniadis, and H. I. Smith, Phys. Rev. Lett. 63, 1894 (1989).

${ }^{11}$ L. I. Glazman, I. M. Ruzin, and B. I. Shklovskii, Phys. Rev. B 45, 8454 (1992); D. V. Averin and K. K. Likharev, in Nanostructures and Mesoscopic Systems, edited by W. P. Kirk and M. A. Reed (Academic, Orlando, in press).

${ }^{12}$ F. M. de Aguiar and D. A. Wharam, Phys. Rev. B 43, 9984 (1991).

${ }^{13}$ J. Mašek and B. Kramer (unpublished).

${ }^{14}$ A. A. M. Staring, H. van Ilouten, C. W. J. Beenakker, and C. T. Foxon, in High Magnetic Fields in Semiconductor Physics III, edited by G. Landwehr (Springer, Berlin, in press).

${ }^{15}$ For a review of theoretical and experimental aspects of quantum transport in semiconductor nanostructures, see C. W. J. Beenakker and H. van Houten, in Solid State Physics, edited by H. Ehrenreich, F. Seitz, and D. Turnbull (Academic, New York, 1991), Vol. 44, p. 1.

${ }^{16}$ U. Meirav, M. A. Kastner, M. Heiblum, and S. J. Wind, Phys. Rev. B 40, 5871 (1989).

${ }^{17}$ C. de Graaf, J. Caro, S. Radelaar, V. Lauer, and K. Heyers, Phys. Rev. B 44, 9072 (1991).

${ }^{18}$ U. Meirav, M. A. Kastner, and S. J. Wind, Phys. Rev. Lett. 65, 771 (1990).

${ }^{19}$ L. P. Kouwenhoven, N. C. van der Vaart, A. T. Johnson, C. J. P. M. Harmans, J. G. Williamson, A. A. M. Staring, and C. T. Foxon, in Proceedings of the German Physical Society Meeting, Festkörperprobleme/Advances in Solid State Physics (Springer, Berlin, in press), Vol. 31.

${ }^{20}$ A. A. M. Staring, J. G. Williamson, H. van Houten, C. W. J. Beenakker, L. P. Kouwenhoven, and C. T. Foxon, Physica B 175, 226 (1991).

${ }^{21}$ P. L. McEuen, E. B. Foxman, U. Meirav, M. A. Kastner, Y. Meir, N. S. Wingreen, and S. J. Wind, Phys. Rev. Lett. 66, 1926 (1991).

${ }^{22}$ C. J. B. Ford, Phys. Scr. (to be published).

${ }^{23}$ H. van Houten, C. W. J. Becnakker, and A. A. M. Staring, in Single Charge Tunneling, edited by $\mathrm{H}$. Grabert and $\mathrm{M}$. H. Devoret (Plenum, New York, in press). This is a recent review of single-electron tunneling in semiconductors.

${ }^{24}$ S. E. Laux, D. J. Frank, and F. Stern, Surf. Sci. 196, 101 (1988).

${ }^{25}$ J. A. Nixon and J. H. Davies, Phys. Rev. B 41, 7929 (1990).

${ }^{26}$ G. Timp, R. Behringer, J. E. Cunningham, and R. E. Howard, Phys. Rev. Lett. 63, 2268 (1989).

${ }^{27}$ A. M. Chang, G. Timp, T. Y. Chang, J. E. Cunningham, P. M. Mankiewich, R. E. Behringer, and R. E. Howard, Solid State Commun. 67, 769 (1988).

${ }^{28}$ R. I. Shekhter, Zh. Eksp. Teor. Fiz. 63, 1410 (1972) [Sov. 
Phys. JETP 36, 747 (1973)].

${ }^{29}$ I. O. Kulik and R. I. Shekhter, Zh. Eksp. Teor. Fiz. 68, 623 (1975) [Sov. Phys. JETP 41, 308 (1975)].

${ }^{30}$ K. Mullen, E. Ben-Jacob, R. C. Jaklevic, and Z. Schuss, Phys. Rev. B 37, 98 (1988); M. Amman, K. Mullen, and E. Ben-Jacob, J. Appl. Phys. 65, 339 (1989).

${ }^{31}$ K. K. Likharev, IBM J. Res. Dev, 32, 144 (1988); D. V. Averin and K. K. Likharev, in Mesoscopic Phenomena in Solids, edited by B. L. Al'tshuler, P. A. Lee, and R. A. Webb (Elsevier, Amsterdam, 1991). This is a comprehensive review of single-electron tunneling in metals.

${ }^{32}$ L. I. Glazman and R. I. Shekhter, J. Phys. Condens. Matter 1, 5811 (1989).

${ }^{33}$ L. D. Landau and E. M. Lifshitz, Electrodynamics of Continuous Media (Pergamon, New York, 1960).

${ }^{34}$ D. V. Averin and A. N. Korotkov, Zh. Eksp. Teor. Fiz. 97, 1661 (1990) [Sov. Phys. JETP 70, 937 (1990)]; A. N. Korotkov, D. V. Averin, and K. K. Likharev, Physica B 165\&166, 927 (1990); D. V. Averin, A. N. Korotkov, and K. K. Likharev, Phys. Rev. B 44, 6199 (1991).

${ }^{35}$ D. V. Averin and A. A. Odintsov, Phys. Lett. A 140, 251 (1989); D. V. Averin and Yu. V. Nazarov, Phys. Rev. Lett. 65, 2446 (1990).

${ }^{36}$ L. I. Glazman and K. A. Matveev, Pis'ma Zh. Eksp. Teor. Fiz. 51, 425 (1990) [JETP Lett. 51, 484 (1990)].

${ }^{37}$ L. I. Glazman (private communication).

${ }^{38}$ A. B. Fowler, A. Hartstein, and R. A. Webb, Phys. Rev. Lett. 48, 196 (1982); R. A. Webb, A. Hartstein, J. J. Wainer, and A. B. Fowler, ibid. 54, 1577 (1985).

${ }^{39}$ R. F. Kwasnick, M. A. Kastner, J. Melngailis, and P. A. Lee, Phys. Rev. Lett. 52, 224 (1984); M. A. Kastner, R. F.
Kwasnick, J. C. Licini, and D. J. Bishop, Phys. Rev. B 36, 8015 (1987).

${ }^{40}$ P. A. Lee, Phys. Rev. Lett. 53, 2042 (1984).

${ }^{41}$ R. K. Kalia, W. Xue, and P. A. Lee, Phys. Rev. Lett. 57, 1615 (1986).

${ }^{42}$ C. J. Adkins, in Hopping and Related Phenomena, edited by H. Fritsche and M. Pollak (World Scientific, New Jersey, 1990).

${ }^{43}$ B. L. Al'tshuler, Pis'ma Zh. Eksp. Teor. Fiz. 41, 530 (1985) [JETP Lett. 41, 648 (1985)].

${ }^{44}$ P. A. Lee and A. D. Stone, Phys. Rev. Lett. 55, 1622 (1985).

${ }^{45}$ A. B. Fowler, G. L. Timp, J. J. Wainer, and R. A. Webb, Phys. Rev. Lett. 57, 138 (1986); A. B. Fowler, J. J. Wainer, and R. A. Webb, IBM J. Res. Dev. 32, 372 (1988).

${ }^{46} \mathrm{M}$. Green and M. Pollak, in Hopping and Related Phenomena, edited by H. Fritsche and M. Pollak (World Scientific, New Jersey, 1990).

${ }^{47}$ B. J. van Wees, L. P. Kouwenhoven, C. J. P. M. Harmans, J. G. Williamson, C. E. Timmering, M. E. I. Broekaart, C. T. Foxon, and J. J. Harris, Phys. Rev. Lett. 62, 2523 (1989).

${ }^{48}$ U. Sivan, Y. Imry, and C. Hartzstein, Phys. Rev. B 39, 1242 (1989).

${ }^{49}$ R. J. Brown, C. G. Smith, M. Pepper, M. J. Kelly, R. Newbury, H. Ahmed, D. G. Hasko, J. E. F. Frost, D. C. Peacock, D. A. Ritchie, and G. A. C. Jones, J. Phys. Condens. Matter 1, 6291 (1989).

${ }^{50}$ D. A. Wharam, M. Pepper, R. Newbury, H. Ahmed, D. G. Hasko, D. C. Peacock, J. E. F. Frost, D. A. Ritchie, and G. A. C. Jones, J. Phys. Condens. Matter 1, 3369 (1989).

${ }^{51}$ J. K. Jain, Phys. Rev. Lett. 60, 2074 (1988). 